\title{
Proposed Activities to Address Regulatory Gaps and Challenges for Licensing Advanced Reactors Using Seismic Isolation
}

Annie M. Kammerer Andrew S. Whittaker Justin L. Coleman

December 2016

The INL is a

U.S. Department of Energy

National Laboratory

operated by

Battelle Energy Alliance

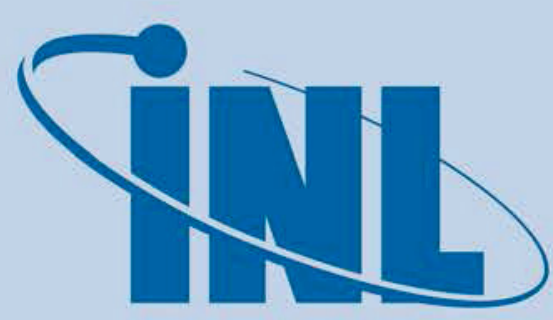

Idaho National Laboratory

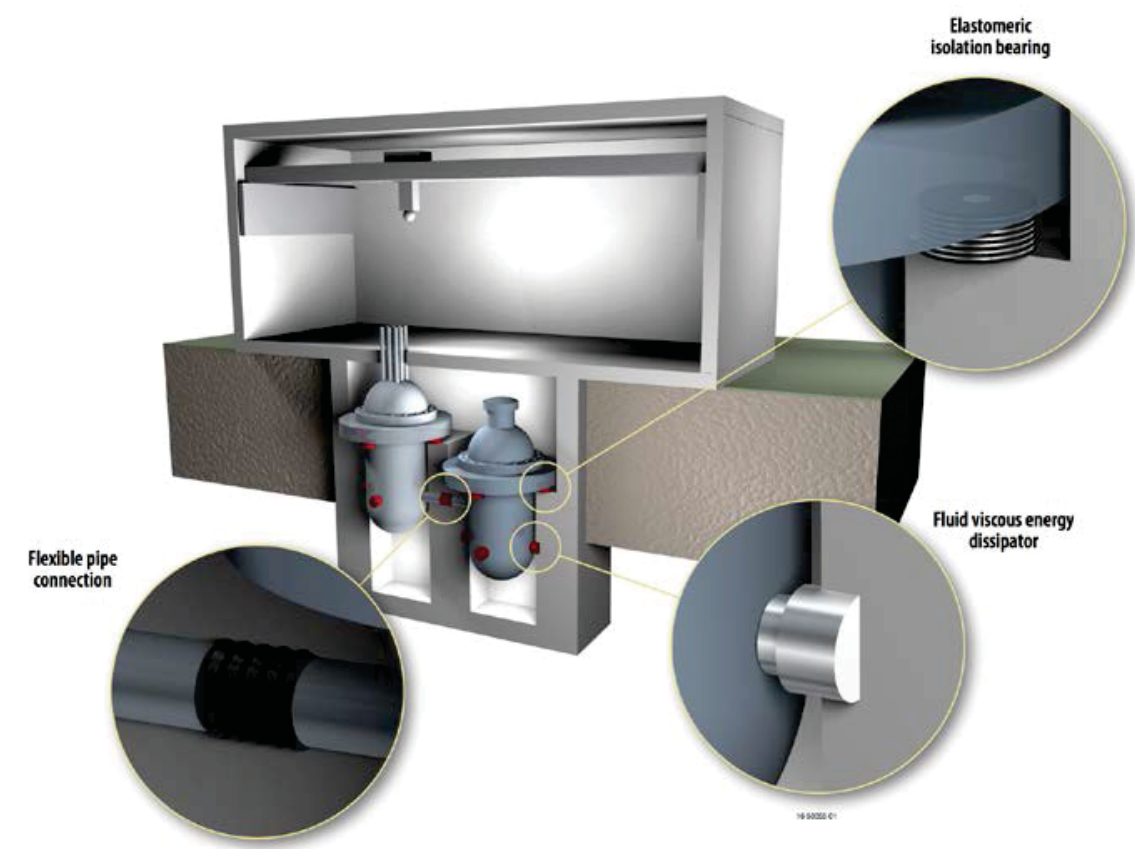




\section{DISCLAIMER}

This information was prepared as an account of work sponsored by an agency of the U.S. Government. Neither the U.S. Government nor any agency thereof, nor any of their employees, makes any warranty, expressed or implied, or assumes any legal liability or responsibility for the accuracy, completeness, or usefulness, of any information, apparatus, product, or process disclosed, or represents that its use would not infringe privately owned rights. References herein to any specific commercial product, process, or service by trade name, trade mark, manufacturer, or otherwise, does not necessarily constitute or imply its endorsement, recommendation, or favoring by the U.S. Government or any agency thereof. The views and opinions of authors expressed herein do not necessarily state or reflect those of the U.S. Government or any agency thereof. 
INL/EXT-16-40668

Revision 0

\title{
Proposed Activities to Address Regulatory Gaps and Challenges for Licensing Advanced Reactors Using Seismic Isolation
}

\author{
Annie M. Kammerer \\ Andrew S. Whittaker \\ Justin L. Coleman
}

December 2016

\begin{abstract}
Idaho National Laboratory
INL ART TDO Program

Idaho Falls, Idaho 83415
\end{abstract}

http://www.inl.gov

Prepared for the

U.S. Department of Energy

Office of Nuclear Energy

Under DOE Idaho Operations Office

Contract DE-AC07-05ID14517 



\section{INL ART TDO Program}

\section{Proposed Activities to Address Regulatory Gaps and Challenges for Licensing Advanced Reactors Using Seismic Isolation}

INL/EXT-16-40668

Revision 0

December 2016

Approved by:
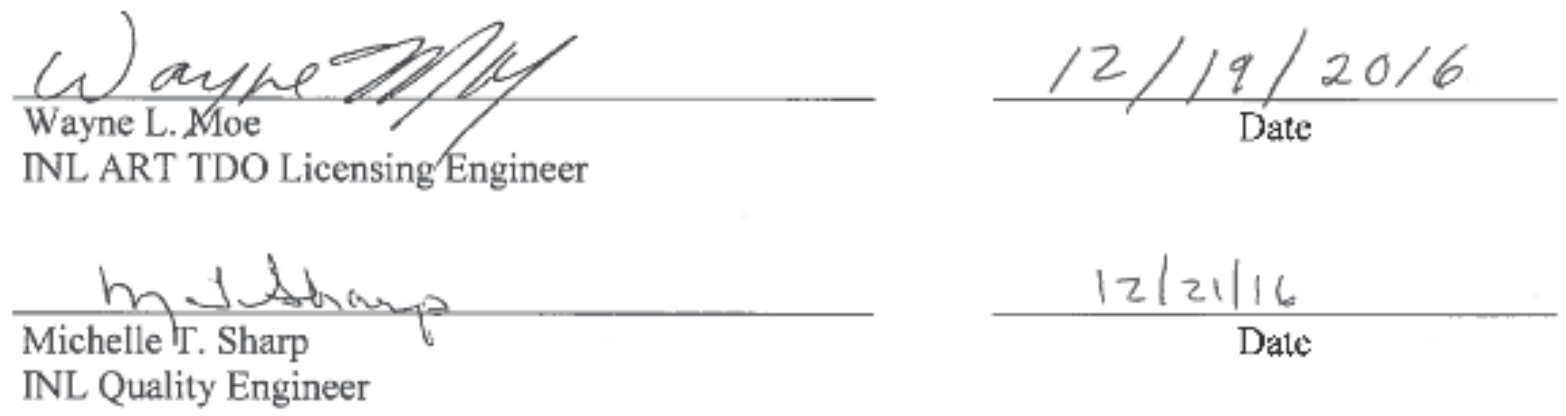



\section{EXECUTIVE SUMMARY}

Over the last decade, particularly since implementation of the certified design regulatory approaches outlined in 10 CFR 52, "Licenses, Certifications, and Approvals for Nuclear Power Plants," interest has been increasing in the use of seismic isolation (SI) technology to support seismic safety in nuclear facilities. In 2009, the United States (U.S.) Nuclear Regulatory Commission (NRC) initiated research activities to develop new guidance targeted at facilities incorporating isolation technology because SI is being considered for nuclear power plants in the U.S. One product of that research, which has been developed around a risk-informed regulatory approach, is a draft Nuclear Regulatory Commission (NUREG) series report that investigates and discusses considerations for use of SI in otherwise traditionally-founded large light water reactors (LWRs). A coordinated effort led to new provisions for SI of LWRs in the American Society of Civil Engineers standard American Society of Civil Engineers/Structural Engineering Institute (ASCE/SEI) 4-16, "Seismic Analysis of Safety-Related Nuclear Structures." The risk-informed design philosophy that underpinned development of the technical basis for these documents led to a set of proposed performance objectives and acceptance criteria intended to serve as the foundation for future NRC guidance on the use of SI and related technology.

Although the guidance provided or expected to be provided in the draft SI NUREG/CR report and ASCE/SEI 4-16 provides a sound basis for further development of nuclear power plant (NPP) designs incorporating SI, these initial documents focused on surface-founded or near-surface-founded LWRs and were, necessarily, limited in scope. For example, there is limited information in both the draft NUREG/CR report and ASCE/SEI 4-16 related to nonlinear analysis of soil-structure systems for deeplyembedded reactors, isolation of components, and use of vertical isolation systems. Also not included in the draft report are special considerations for licensing of isolated facilities using the certified design approach in 10 CFR 52 or a detailed discussion of seismic probabilistic risk assessments for isolated facilities.

To identify and address limitations in the initial guidance, Idaho National Laboratory (INL) has initiated several projects focused on further developing the technical and licensing underpinnings for facilities using SI technology. These efforts include a 2014 workshop focused on SI (Coleman and Sabharwall 2014), development of new structural analysis tools and methodologies appropriate for SI, and development of INL report INL/EXT-15-36945, "Regulatory Gaps and Challenges for Licensing Advanced Reactors Using Seismic Isolation," (Kammerer, Whittaker, and Coleman 2016) that identified and described regulatory guidance gaps and challenges related to licensing of advanced reactors using SI. Nearly all of the gaps and challenges identified in INL/EXT-15-36945 fall outside the scope of current research and development efforts (including those at INL). This report builds on information in INL/EXT-15-36945 by providing additional actionable details related to the scope and possible schedule of activities to address the gaps and challenges identified. Although some discussions and issues have been updated or revised in this report as a result of peer review and feedback from industry stakeholders, this report is intended to supplement, and not replace, the earlier report.

Because efforts to date related to regulatory guidance development (e.g., the draft NRC SI NUREG/CR report) have principally considered designs similar to the light water reactor technologies currently being licensed, the existing literature (as discussed in INL/EXT-15-36945) is reflective of traditional LWR designs. All of the regulatory guidance gaps and challenges that apply to large surfacefounded LWRs also apply to advanced reactors; and the LWR case often provides a simplified example as compared to the range of cases found in advanced reactors. Advanced reactor designs also lead to new gaps and challenges not faced in LWR design. Although both this report and INL/EXT-15-36945 discuss advanced reactors broadly ${ }^{1}$, the exact set of challenges and potential solutions for any particular reactor

\footnotetext{
${ }^{1}$ Here the term advanced reactor applies to any NPP design other than large surface-founded LWRs.
} 
design is technology-specific. The activities detailed in this report necessarily require some level of specificity. However, significant effort is made to develop the activities to be as technology neutral as possible.

Advanced reactors will likely be designed and constructed very differently from LWRs, regardless of whether they employ SI and damping devices. Key technical advances in civil and structural engineering needed to deploy advanced reactors are:

1. Development of performance-based seismic design and assessment procedures for non-LWR reactors

2. Development and deployment of analysis methodologies suitable for computing the response of deeply-embedded power reactors, including nonlinear time domain, soil-structure-interaction analysis

3. Development, prototyping, and deployment of two-dimensional and three-dimensional isolation systems suitable for components ranging in size and complexity from diesel generators to reactor vessels.

Advances in Items 1 and 2, above, are needed for deeply-founded NPPs, regardless of whether seismic protective measures (i.e., such as those noted in Item 3) are deployed. A focus on developing additional guidance for isolation of major components is enhanced in this report (as compared to the discussion in INL/EXT-15-36945) due to input provided during the technical peer review process. Not covered in this report are other important technical advances in civil and structural engineering needed for economical deployment of advanced reactors (e.g., development and deployment of modular construction strategies used to minimize "one-off" fieldwork, schedule delays, and construction cost).

In addition to improving safety, SI offers potentially significant economic benefits for advanced reactors because the isolation system can reduce site-dependent seismic demands below pre-qualified levels in certified design. Their protective isolation systems would be site-specific and based on sitespecific ground motions. Although design optimization and commercial aspects related to the use of SI have been identified in Coleman and Sabharwall (2014) and elsewhere as possible issues or areas of opportunity, only topics that may impact efficient licensing are addressed in this report.

INL/EXT-15-36945 identifies gaps and challenges that are discussed throughout that report and summarized in Section 7. INL/EXT-15-36945 also identifies critical and high-impact/high-value topics that should be addressed in the short term and that must be satisfactorily completed before substantial progress on other tasks can be made. This report builds upon INL/EXT-15-36945 by providing a more detailed roadmap that prioritizes and organizes activities. The prioritization considers the following elements:

- The potential level of regulatory risk

- The potential for the work to provide high-impact and high-value in terms of supporting efficient licensing activities

- The sequencing of the issue to resolving other gaps and challenges identified.

The gaps and challenges identified are summarized in Table 2. The 19 unique gaps and challenges identified and evaluated have each been assigned a Topic number; and tasks needed to address an issue are numbered using the associated Topic numbers. Table 2 provides a summary roadmap of identified tasks, including possible timelines. Based on the roadmap provided, it is recommended that four Topics should be prioritized for funding:

- Development of NLSSI tools and guidance, including verification and validation activities. (Topic 1)

- Development of SPRA methodologies for seismically isolated facilities, with an emphasis on advancement of methods applicable when linear scaling assumptions do not apply. (Topic 6) 
- Clarification of the approaches to licensing of facilities using seismic isolation technology within the certified design process and the associated clarification of the intent of the term "foundation" in existing requirements and guidance (Topics 12 and 14)

- Development of approaches and guidance for isolation of large equipment (Topic 4)

One DOE goal specified in the draft document entitled, "Vision and Strategy for the Development and Deployment of Advanced Reactors," ${ }^{2}$ is:

"By the early 2030s, at least two non-light water advanced reactor concepts would have reached technical maturity, demonstrated safety and economic benefits, and completed licensing reviews by the U.S. Nuclear Regulatory Commission (NRC) sufficient to allow construction to go forward."

SI has been identified as an important technology that both facilitates the siting of advanced reactors in a wider range of locations and improves seismic safety. SI can also minimize the need for "one-off" advanced reactor designs that would need to be changed to meet site-specific seismic hazard curves. Minimization of one-off designs would improve economics of advanced reactors. Several of the topics and tasks identified in this report support the design and licensing of advanced reactors, regardless of whether SI technology is used, particularly if the NPPs are deeply embedded. Additionally, current advanced reactor venders, in collaboration with INL, are interested in initiating sensitivity studies for seismic isolation of their specific technologies. This report provides a 10 -year timeline for completing activities to address the issues identified. Not starting the four near term activities in a timely way would limit the impact of the collaborative work with advanced reactor venders due to the regulatory uncertainty. A delayed start, as well as a lengthened timeline due to insufficient funding, would also make achieving the goals laid out in the DOE vision document less likely.

\footnotetext{
2 The 27 May 2016 version of this document is available for download at https://www.energy.gov/ne/downloads/draft-visionand-strategy-development-and-deployment-advanced-reactors.
} 


\section{ACKNOWLEDGEMENTS}

The Advanced Reactor Technologies program commissioned this report. INL's Seismic Research Group, which conducts research on nuclear applications covering, among other topics, the use of non-linear (time domain) soil-structure-interaction analysis, provided input. INL Seismic Advisory Group, composed of Dr. Robert Kennedy (RPK Structural Mechanics Consulting), Dr. Nilesh Chokshi (NCChokshi Consulting), Dr. Robert Budnitz (Lawrence Berkeley National Laboratory, LBNL), and Dr. Farhang Ostadan (Bechtel), reviewed this report, including the proposed tasks and prioritization. Dr. Michael Salmon (Los Alamos National Laboratory, Prof. Youssef Hashash (University of Illinois), and John Richards (EPRI) also provided input.

This work benefited from input by industry stakeholders at a June 2016 workshop and through the INL-TerraPower CRADA activities.

The work that underpins this report benefits from input from a broad research review panel that provided review and advice on a suite of coordinated NRC-supported projects at LBNL aimed at improving the regulation of seismic safety for current large light water reactors. One of those earlier NRC-supported projects, led by Prof. Andrew Whittaker (SUNY-Buffalo), developed the draft NRC SI NUREG/CR report. The earlier suite of research projects at LBNL was led by Dr. Robert Budnitz of LBNL. The research review group was composed of Dr. Robert Kennedy (RPK Structural Mechanics Consulting), Dr. Nilesh Chokshi (NCChokshi Consulting and NRC [retired]), Dr. Donald Moore (Southern Nuclear Operating Company [retired]), Dr. James Johnson (James J. Johnson and Associates), Mr. Antonio Godoy (James J. Johnson and Associates and International Atomic Energy Agency [retired]), and Prof. Jonathan Stewart (University of California, Los Angeles). Dr. Robert Kennedy (RPK Structural Mechanics Consulting) chaired that research review group. 


\section{CONTENTS}

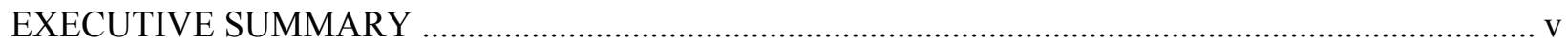

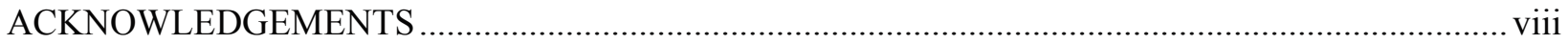

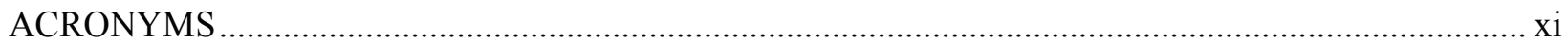

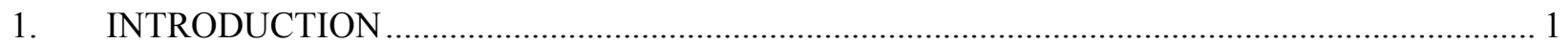

2. REGULATORY CHALLENGES AND GAPS RELATED TO ENGINEERING TOOLS

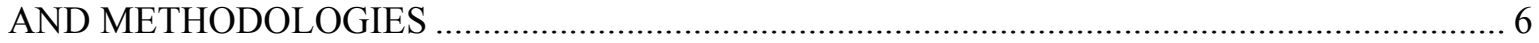

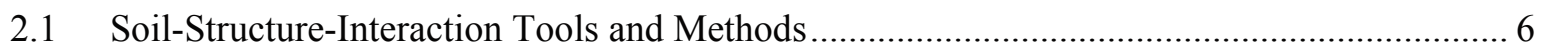

2.1.1 Development of Nonlinear Soil-Structure-Interaction Tools and Methods ................. 6

2.1.2 Assessment of Application and Limitations of 1-Dimentional Assumption in Site Response Analysis .......................................................................................... 9

2.1.3 Ground Motion Selection, Modification and Horizon Definition............................. 10

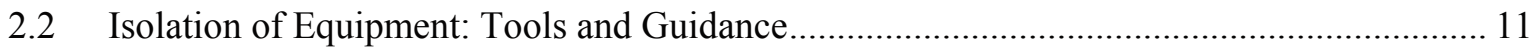

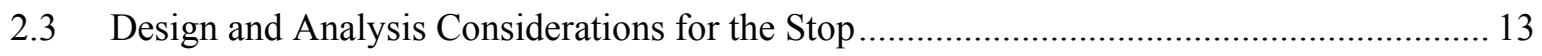

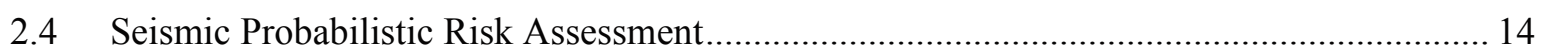

2.4.1 Seismic Probabilistic Risk Assessment Calculations for Failure of the

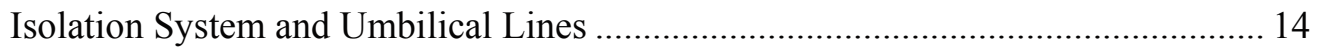

2.4.2 Seismic Probabilistic Risk Assessment Calculations for Isolated

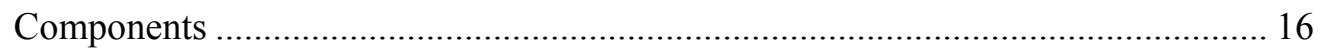

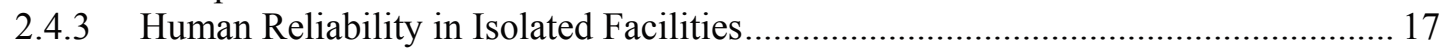

2.4.4 Guidance for Seismic Probabilistic Risk Assessment Feedback to Design ............... 17

2.4.5 Generic HCLPF Capacity Tables for Isolated Nuclear Power Plants ........................ 18

3. CHALLENGES AND GAPS RELATED TO REGULATORY APPROACHES AND TERMINOLOGY

3.1 Terminology and Approach for Licensing Isolated Nuclear Power Plants Using the Certified Design Process 20

3.2 Challenges and Considerations Related to Vertical Loading of Certified Designs

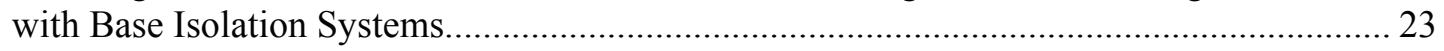

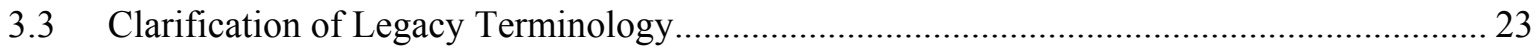

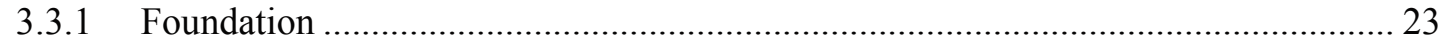

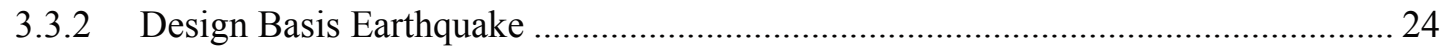

4. CONFIGURATIONS AND APPLICATIONS NOT ADDRESSED IN FORTHCOMING

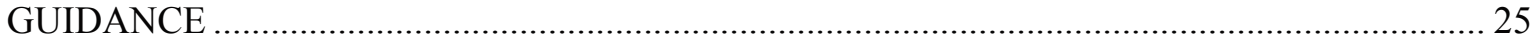

4.1 Use of Isolators Exposed to Radiation and Other Environmental Conditions

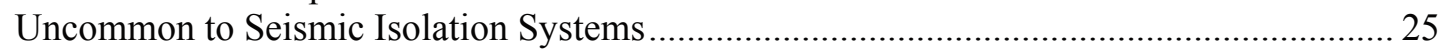

4.2 Isolation of Components, Equipment, and Distributed Systems ........................................ 25

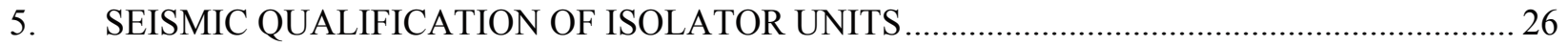

6. LICENSING COMMITMENTS FOR CONSTRUCTION AND OPERATIONS ......................... 27

6.1 Considerations for Inspection, Testing, Analysis, and Acceptance Criteria ......................... 27 
6.2 In-Service Inspections, Testing, and Operations...................................................... 27

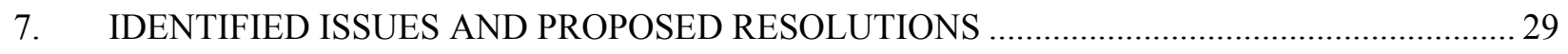

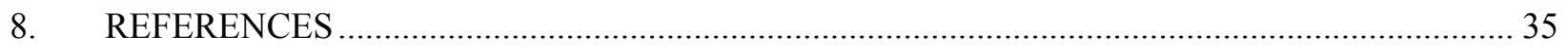

\section{FIGURES}

Figure 1. Seismic isolation of a light water reactor (ASCE 2016) ........................................................ 2

Figure 2. Deeply-embedded advanced reactor incorporating seismic protective systems........................16

Figure 3. Approaches for comparing site-independent certified seismic design response spectrum against site-dependent foundation input response spectrum (modified for clarity from

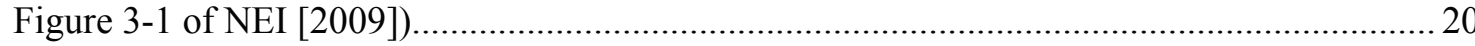

Figure 4. Diagram showing potential comparison horizon for evaluation of site against certified design for surface-founded, base-isolated nuclear power plant.

\section{TABLES}

Table 1. Definitions of proposed terms for use with seismically-isolated certified designs..................... 22

Table 2. Summary of identified topics, importance, and proposed resolutions. ......................................... 31 


\section{ACRONYMS}

1D one-dimensional

2D two-dimensional

3D three-dimensional

ART Advanced Reactor Technologies

ASCE/SEI American Society of Civil Engineers/Structural Engineering Institute

ASME/ANS American Society of Mechanical Engineers/American Nuclear Society

BDB beyond design basis

CGD commercial-grade dedication

CFR Code of Federal Regulations

CRADA cooperative research and development agreement

CSDRS certified seismic design response spectra

DOE Department of Energy

DRM domain reduction method

EPRI Electric Power Research Institute

FIRS foundation input response spectra

FP Friction Pendulum ${ }^{\mathrm{TM}}$

GMRS ground motion response spectra

GMSM ground motion selection and modification

HCLPF high-confidence of a low-capacity of failure

INL Idaho National Laboratory

ISG Interim Staff Guidance

ITAAC inspection, testing, analysis, and acceptance criteria

LBNL Lawrence Berkeley National Laboratory

LWR light water reactor

MOOSE Multi-Physics Object-Oriented Simulation Environment

NIST National Institute of Science and Technology

NLSSI nonlinear soil-structure interaction

NPP nuclear power plant

NRC Nuclear Regulatory Commission

NUREG/CR NUclear REGulatory Commission contractor report

PGA peak ground acceleration

PSHA probabilistic seismic hazard analysis

R\&D research and development 
RG Regulatory Guide

SCEC Southern California Earthquake Center

$\mathrm{SI}$ seismic isolation

SI-CSDRS seismically-isolated certified seismic design response spectrum

SI-TFRS seismically-isolated top of foundation response spectrum

SIDRS seismic isolation design response spectra

SPRA seismic probabilistic risk assessment

SRA site response analysis

SRP Standard Review Plan (NUREG-0800)

SSC structure, system, and component

SSI soil-structure-interaction

U.S. United States

$\mathrm{V} \& \mathrm{~V} \quad$ verification and validation

$\mathrm{V} / \mathrm{H} \quad$ vertical-to-horizontal (ratio) 


\section{INTRODUCTION}

Advanced nuclear reactors will likely be different from existing large light water reactors (LWRs) in many key safety design approach aspects, such as: reactor technology and operation; passive safety systems; and design and construction of structures, systems, and components (SSCs). Importantly, safety-related SSCs in the advanced reactor concepts currently supported by Department of Energy (DOE) research and development (R\&D) are evolving designs that will likely be housed in reinforced concrete structures that are deeply embedded, which poses both a series of benefits and a corresponding series of challenges in terms of seismic analysis, design, construction, and risk assessment. DOE's Advanced Reactor Technologies (ART) Program commissioned this report as part of a coordinated effort to identify and address potential regulatory challenges related to advanced reactor designs.

Seismic isolation (SI) has developed a significant track record as a result of decades of experience in tens of thousands of structures globally, including a significant number that have been subjected to large seismic ground motions. As a result, SI is considered a viable, practical technology for protection of safety-related SSCs in nuclear facilities. SI is expected to be deployed because it provides benefits related to increased earthquake safety and/or reduced construction costs and potential benefits within the certified design process. The American Society of Civil Engineers (ASCE) and United States (U.S.) Nuclear Regulatory Commission (NRC) have implemented methods of analysis and design for isolation of LWRs either in published or in soon-to-be-published standards and guidance. The stated focus of Chapter 12 of Standard ASCE/SEI 4-16 (ASCE 2016) and of a draft NRC NUREG series (NUREG/CR) report (Kammerer, Whittaker, and Constantinou 2017) ${ }^{3}$ is the horizontal isolation of large near-surface-founded nuclear facilities, which is due in part to the interest shown by industry in the mid-to-late 2000s to deploy SI in nuclear power plants (NPPs) in the U.S.

Early work investigating the implementation of SI in NPPs assumed (for simplicity) cases where seismic input is at a single level across the footprint of the reactor building (i.e., at the foundation level of the isolated LWR, as shown in Figure 1). Some of the tools, techniques, and hardware developed for SI of NPPs with configurations like that shown in Figure 1 would also be applicable, in principal, to deeply-embedded nuclear facilities. However, some new methods of seismic analysis, design, construction, and risk assessment will be required for advanced reactors. These new methods bring with them regulatory guidance gaps and challenges that Idaho National Laboratory (INL) report INL/EXT-1536945, "Regulatory Gaps and Challenges for Licensing Advanced Reactors Using Seismic Isolation," (Kammerer, Whittaker, and Coleman 2016) identified. Many of the gaps identified in that report apply to deeply-embedded reactors, regardless of whether or not they employ SI. Importantly, technical approaches have been identified to address all of the gaps and challenges. Indeed, although some issues require additional $\mathrm{R} \& \mathrm{D}$, several issues that were identified as representing high regulatory risk can be addressed in the short term by developing a technical basis using existing information.

Section 1 of INL/EXT-15-36945 provided a summary of recent advances in the application of seismic isolation to NPPs. It also provided a summary of a draft SI NUREG/CR report that the NRC developed as a result of a research program initiated in 2009 to develop new guidance targeted at facilities using seismic isolation technologies. The guidance in the draft NUREG/CR was incorporated into Chapter 12 of ASCE/SEI 4-16, which is also discussed in INL/EXT-15-36945. These summaries are not repeated here, and the reader should refer to INL/EXT-15-36945 for more information.

\footnotetext{
${ }^{3}$ Some of the content in this report is based on the draft NUREG/CR document. If the NUREG/CR content is significantly revised or changed before issue, discussions and conclusions in this report may need to be evaluated and revised.
} 
The guidance expected to be provided in the draft SI NUREG/CR report and ASCE/SEI 4-16 provides a solid technical basis for further development of NPP designs incorporating SI. However, these initial documents focused on surface-founded or near-surface-founded large LWRs and were, necessarily, limited in scope. For example, there is little information in either document related to vertical isolation systems, the isolation of individual systems or components, or the isolation of deeply-embedded reactors and other types of nuclear facilities (e.g., small modular rectors, high-temperature gas reactors, or fast reactors). Also missing from the draft SI NUREG/CR report are unique considerations both for licensing isolated facilities using the certified design approach in the Code of Federal Regulations (10 CFR 52) ${ }^{4}$ and for performing seismic probabilistic risk assessments (SPRAs) for isolated facilities.

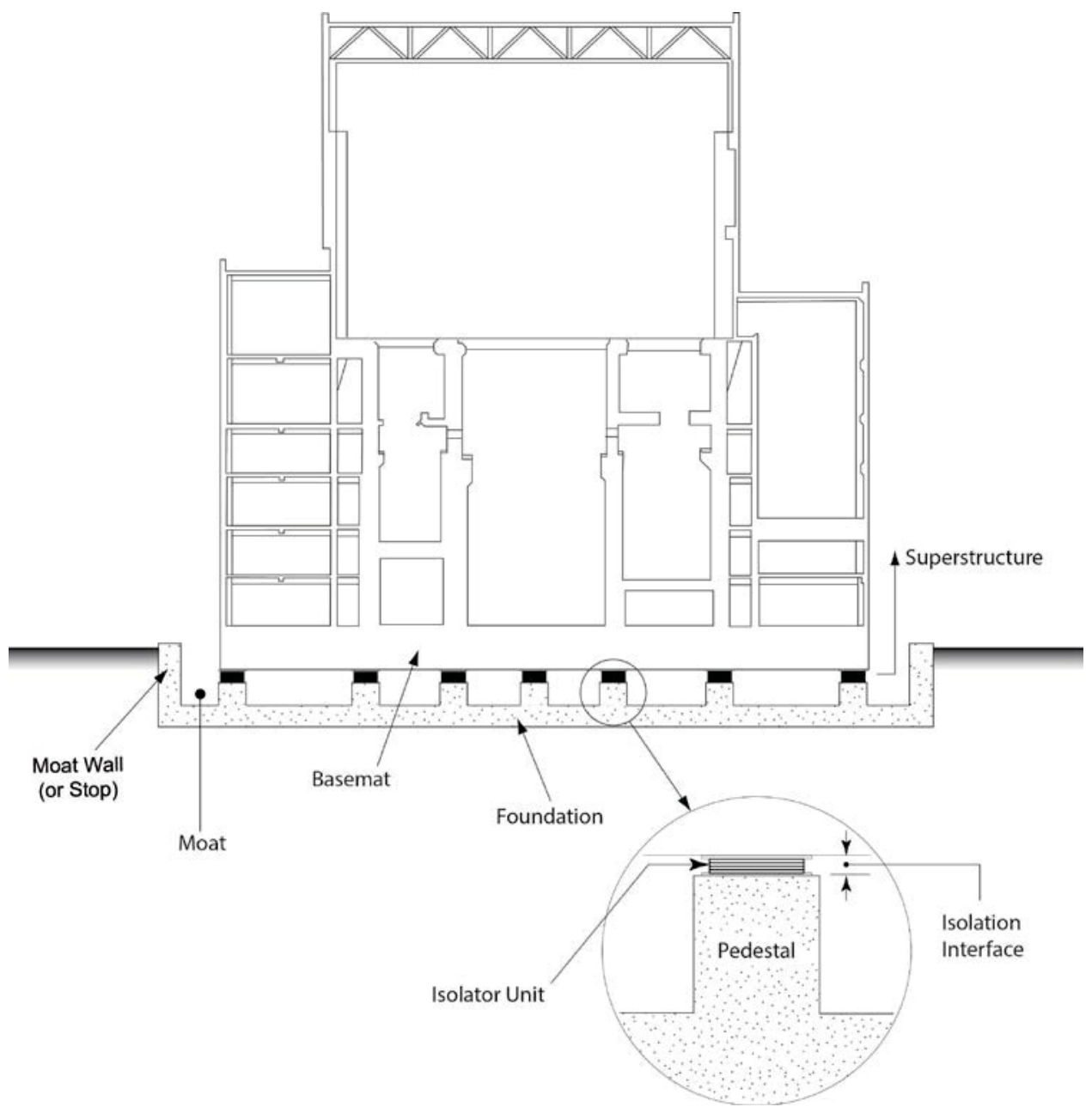

Figure 1. Seismic isolation of a light water reactor (ASCE 2016).

To identify and address the limitations in the existing guidance, INL initiated several projects focused on further developing technical and licensing underpinnings for facilities using SI technology. In August 2014, INL sponsored a workshop that was attended by representatives from DOE, national laboratories, industry, the Electric Power Research Institute (EPRI), NRC, and academia. INL report INL/EXT-14-33234 (Coleman and Sabharwall 2014) documented the workshop.

\footnotetext{
${ }^{4}$ The remainder of the issues and tasks included here apply equally to 10 CFR Part 50 and Part 52.
} 
More recently, DOE's ART group sponsored a report to identify regulatory impediments to the efficient licensing of advance reactors using SI technology. This resulted in INL/EXT-15-36945 (Kammerer, Whittaker, and Coleman 2016), which identified and discussed regulatory guidance gaps and challenges, and included a summary in Section 7. INL/EXT-15-36945 also identified important highimpact and high-value topics that should be addressed in the short term and that need to be satisfactorily completed before substantial progress on other tasks can be made.

DOE's ART group also commissioned this follow-up report, which builds upon the information in INL/EXT-15-36945 by further detailing activities (and initially estimating associated time and resources) needed to address the gaps and challenges identified. Although some discussions and issues have been updated or revised in this report as a result of peer review and feedback from industry stakeholders, this report is intended to supplement, not replace, the earlier report.

The gaps and challenges identified are summarized in Table 2. The 19 unique gaps and challenges identified and evaluated have each been assigned a Topic number; and proposed actions are assigned an associated task number. Table 2 provides a summary roadmap of identified tasks, including possible timelines. Based on the roadmap provided, it is recommended that four Topics should be prioritized for funding:

- Development of NLSSI tools and guidance, including verification and validation activities. (Topic 1)

- Development of SPRA methodologies for seismically isolated facilities, with an emphasis on advancement of methods applicable when linear scaling assumptions do not apply. (Topic 6)

- Clarification of the approaches to licensing of facilities using seismic isolation technology within the certified design process and the associated clarification of the intent of the term "foundation" in existing requirements and guidance (Topics 12 and 14)

- Development of approaches and guidance for isolation of large equipment (Topic 4)

One DOE goal specified in the draft document entitled, "Vision and Strategy for the Development and Deployment of Advanced Reactors," is:

\section{"By the early 2030s, at least two non-light water advanced reactor concepts would have reached technical maturity, demonstrated safety and economic benefits, and completed licensing reviews by the U.S. Nuclear Regulatory Commission (NRC) sufficient to allow construction to go forward."}

SI has been identified as an important technology that both facilitates the siting of advanced reactors in a wider range of locations and improves seismic safety. SI can also minimize one off advanced reactor designs that would otherwise need to be changed to meet site specific seismic hazard curves. Minimization of "one-off" designs should improve economics of advanced reactors. Several of the topics and tasks identified in this report support the design and licensing of advanced reactors, regardless of whether SI technology is used, particularly if the NPPs are deeply embedded. Additionally, current advanced reactor venders, in collaboration with INL, are interested in initiating sensitivity studies for seismic isolation of their specific technologies. This report provides a 10-year timeline for completing activities to address the issues identified. Not starting the four near term activities in a timely way would limit the impact of the collaborative work with advanced reactor venders due to the regulatory uncertainty. A delayed start, as well as a lengthened timeline due to insufficient funding, would also make achieving the goals laid out in the DOE vision document less likely.

in this report provides a detailed roadmap that prioritizes and organizes activities. Table 2 summarizes gaps and challenges and provides information on possible timelines and resource needs. The 19 unique gaps and challenges identified and evaluated have each been assigned a Topic number; and proposed actions are assigned an associated task number.

The prioritization in Table 2 considers the following elements: 
- The potential level of regulatory risk

- The potential for the work to provide high-impact/high-value

- The sequencing of the issue to resolving other gaps and challenges identified.

Additionally, INL and the MCEER at the University at Buffalo, State University of New York, are currently working with TerraPower, LLC, through a cooperative research and development agreement $(\mathrm{CRADA})^{5}$ to develop a methodology for evaluating the potential benefits of SI and to support advances in the associated numerical modeling tools and approaches. A focus of the CRADA is to couple a nonlinear soil-structure-interaction (NLSSI) methodology with advanced nonlinear numerical models of isolator units and fluid-structure interaction. The activities described in this report benefitted from industry feedback associated with the ongoing CRADA efforts.

Activities to address nearly all of the regulatory guidance gaps and challenges identified in INL/EXT15-36945 lie outside the scope of current technology R\&D efforts at INL and elsewhere. These gaps and challenges relate to seismic design of isolated and non-isolated advanced reactors and their likely internal components. To achieve its goal, INL/EXT-15-36945 addressed three general categories of topics:

1. Gaps and challenges associated with SI of near-surface-founded reactors

2. Gaps and challenges associated with deeply-embedded reactors ${ }^{6}$

3. Gaps and challenges associated with isolation of SSCs.

This report provides a more detailed roadmap to the $R \& D$ activities required to close or overcome all three sets of gaps and challenges; and the products of the R\&D work conducted to close the identified gaps and challenges should be moved quickly into regulatory space prior to commercial deployment. All of the gaps and challenges identified can ultimately be addressed through new guidance (in some cases, after work to develop a clear and supportable technical basis is conducted). An achievable path forward has been described for all gaps and challenges previously identified in INL/EXT-15-36945.

In both INL/EXT-15-36945 and this report, a gap is defined as a topic that has not been directly addressed in current regulatory guidance. By contrast, a challenge is defined as a topic for which the existing guidance creates ambiguities or conflicts and needs to be augmented. In some cases, the challenges arise because the existing guidance assumes the use of a specific tool (e.g., the structural modeling tool SASSI [Ostadan and Deng 2011]) or methodology or because the guidance is focused on a specific NPP technology (e.g., LWRs). Some challenges relate to traditional terminology and arise as a result of the more complex foundation system associated with a base-isolated superstructure.

In the U.S. regulatory framework, the Code of Federal Regulations (CFR) generally provides high-level requirements targeted on performance objectives. NPP performance must be assessed against the objectives and demonstrated to be adequate and reliable with respect to the intended safety function. Indirect deterministic methods (with a probabilistic basis) and prescriptive details, such as those provided in Standard ASCE/SEI 43-05 (ASCE 2005), have been used for new LWRs to achieve performance objectives. Advanced reactor technologies will focus on achieving safety performance objectives more directly, using risk-informed, performance-based methods, placing less reliance on the deterministic methods and prescriptive details currently being used. The indirect methods and prescriptive design approaches used in the past have been described in codes, standards and guidance documents, rather than the CFR. The NRC recently published NUREG/CR-7214 (Budnitz and Mieler 2016), which provides insights into possible new and more direct approaches to demonstrating that risk targets are met.

The point-of-contact for the CRADA is Justin Coleman, an author of this report.

6 All of the items in (1) also apply to isolated deeply-embedded reactors and many apply to non-isolated deeply-embedded reactors. 
Guidance has also been developed by INL (2014) and the American Society of Mechanical Engineers/American Nuclear Society (ASME/ANS 2013).

Because the earlier efforts developed regulatory guidance (e.g., the draft SI NUREG/CR report) focused on light water reactor technologies similar to those being licensed at the time, the existing literature is reflective of traditional LWR designs (see examples in INL/EXT-15-36945). As noted above, all of the regulatory guidance gaps and challenges that apply to LWRs and LWR-related guidance also apply to advanced reactors. Often LWR case studies provide a simplified example as compared to the varieties of configuration and more direct risk-based safety approaches found in advanced reactors. Thus, advanced reactor designs often lead to new gaps and challenges not faced in LWR design. Although INL/EXT-15-36945 and this report discuss advanced reactors broadly, the exact set of challenges and potential solutions for any particular reactor design will be technology-specific.

Section 2 of this report describes regulatory challenges and gaps associated with tools and methodologies applied to the seismic design and evaluation of safety-related SSCs. Section 3 discusses a unique set of regulatory challenges and gaps associated specifically with the certified design licensing process of 10 CFR Part 52. Section 3 also provides discussion aimed at clarifying legacy terminology that is ambiguous when applied to NPPs using SI technology as part of foundation design. Section 4 addresses facility configurations and applications not addressed in the draft SI NUREG/CR report. Section 5 presents a proposed approach to seismic qualification of the site-specific isolator units consistent with ASCE/SEI 4-16 and the draft SI NUREG/CR report. Section 6 addresses considerations for licensing commitments for construction and operations specific to an isolated NPP. Section 7 summarizes this report and identifies key issues for consideration and possible resolution. Throughout the remainder of the document, the issues discussed are also identified by topic number to allow for clearer tracking of the issues and tasks in Table 2 which provides a detailed program timeline for addressing the gaps and challenges identified.

A peer-review process enhanced and refined the prioritization and proposed activities described herein through a workshop attended by the INL Seismic Advisory Group and other stakeholder participants, including vendors considering the isolated designs. For this reason, the discussion of the issues, approaches for resolution, and prioritization do not map directly with INL/EXT-15-36945. 


\section{REGULATORY CHALLENGES AND GAPS RELATED TO ENGINEERING TOOLS AND METHODOLOGIES}

\subsection{Soil-Structure-Interaction Tools and Methods}

\subsubsection{Development of Nonlinear Soil-Structure-Interaction Tools and Methods}

As discussed in detail in Section 2.1.1 of INL/EXT-15-36945, equivalent linear site response analysis (SRA) and soil-structure-interaction (SSI) analysis are commonplace in the nuclear power industry at the time of this writing. Equivalent linear analysis, which utilizes secant stiffness and equivalent viscous damping, is likely sufficiently accurate for the purposes of design and risk assessment if soil strains are small and there is no localized relative movement (i.e., gapping, sliding, and uplift) between the foundation and the adjacent soil. Larger strains may be expected for weaker or more flexible soils at sites in the U.S. that experience shaking at the design basis level and greater. This includes shaking at the NPP beyond-design-basis (BDB) ground motion levels considered in an SPRA.

Although its use is widespread, neither the CFR nor NRC regulatory guidance requires that equivalent linear analysis tools be used for SSI analysis. However, as a result of the long and substantial history, the use of equivalent linear tools is assumed in a number of current NRC guidance documents, as described in INL/EXT-15-36945. This represents a challenge to the efficient licensing of both new NPP designs using SI and new NPP designs that are deeply embedded (regardless of whether they are designed with SI or not).

However, as discussed in INL/EXT-15-36945, recent documentation issued by the NRC (2013) for the mPower reactor (www.generationmpower.com) appears to recognize the special challenges and needs related to SSI analysis approaches for deeply-embedded reactor designs. Some of the aspects unique to deeply-embedded ${ }^{7}$ structures that the NRC identified as needing further investigation are:

- Geometric nonlinear behavior such as separation (or gapping) and sliding between the soil and structure

- Nonlinear dynamic effects in the soil, such as dynamic changes in hydrostatic pressure and shear strain, which affect energy dissipation

- The extent to which non-vertically propagating shear waves (including surface waves) may be more important for deeply-embedded structures than for those with shallow embedment.

Some of these aspects of seismic response in NPPs may also be important for surface- and near-surface-founded structures. As described in INL/EXT-15-36945, SSI behaviors that may not be well captured by linear equivalent tools include nonlinear deformation in the soil and/or supported foundation and structure, relative movement along the horizontal soil-foundation interface (termed sliding), localized relative horizontal movement of the foundation with respect to the adjacent soil (gapping) and large vertical movement of the foundation (termed gapping) with respect to the adjacent soil. Vertical uplift may also occur in extreme cases.

The appropriate approach to addressing the above challenge to efficient design and licensing of advanced reactors is focused on developing tools, methods, and guidance to be used in the nuclear industry. These tools, methods and guidance can be developed by expanding and enhancing resources already available at INL and in other institutions. Efforts to appropriately model gapping, sliding, and

There is no single definition of deeply embedded. Herein, deeply embedded is used to describe those nuclear facilities that experience seismic input at multiple foundation levels and/or over the height of the embedded perimeter so that response of SSCs depart substantially from those computed assuming seismic input at the lowest foundation level only. Accordingly, deeply embedded is a function of facility geometry, contents, and soil types, mechanical properties, and layering. 
uplift and to investigate their potential impact on SSC response and facility risk, have been undertaken at INL as part of a research program focused on advanced SPRAs.

A nonlinear time-domain code for performing NLSSI simulations, Mastodon, ${ }^{8}$ is being developed using the NQA-1 (ASME 2015) certified INL Multi-Physics Object-Oriented Simulation Environment (MOOSE) framework (see Coleman et al. 2016b). INL efforts focus on commercially-supported codes that meet NQA-1 requirements and have formal version control, both of which are generally required for applications in the nuclear industry in the U.S. These codes, once verified and validated for site response analysis (SRA) and SSI analysis by performing tasks 1-1 to 1-9 described below, could explicitly address the three NRC-identified bulleted items above.

In addition to design-related needs, technical needs related to the SPRA (which requires accurate modeling of behavior at the design basis and BDB ground motion levels) and implementation of technologies (such as SI and nonlinear damping devices) have spurred investigation, development, and early deployment of numerical tools for nonlinear (time domain) SSI analysis of NPPs (e.g., Jeremić et al. 2009, 2013; Willford et al. 2010; Bolisetti et al. 2014, 2015; Bolisetti, Whittaker, and Coleman 2016; Coleman et al. 2016a). These studies and the nuclear-community interest in nonlinear time-domain analysis prompted writing of the non-mandatory Appendix B in ASCE/SEI 4-16. The provisions in this appendix are written at a high level in recognition of the state-of-knowledge and state-of-acceptance of nonlinear time domain SSI analysis in the nuclear community. More specificity and design examples are needed to enable straightforward regulation and widespread acceptance. Addressing this need is a planned activity for the next update of Standard ASCE/SEI 4, planned for 2021.

Topic 1, which addresses the lack of verified and validated tools for NLSSI, is considered a high priority, long-term effort. Listed below are technical research, development, and deployment activities that are currently needed to address Topic 1 and will result in a version of Mastodon that meets verification and validation $(\mathrm{V} \& \mathrm{~V})$ requirements. The timeline and resource needs associated with these activities are provided in Table 2 .

Most of the needed actions detailed below are also directly applicable to needs associated with the use of equivalent linear SSI analysis using SHAKE and SASSI. ${ }^{9}$ All are needed to support deployment of deeply-embedded advanced reactors by ensuring that design tools can accurately estimate seismic demands and appropriately support design and risk assessment activities. By coupling activities with ground motion selection and modification (GMSM)-related work (discussed in Section 2.1.3) these activities could directly address issues related to SRA/SSI compatibility, as discussed in Section 2.1.4 of INL/EXT-15-36945. To enable widespread and speedy dissemination, all research-oriented products would be made available via the INL Seismic Research Group website ${ }^{10}$, which is used by this group to disseminate publicly available information.

Addressing Topic 1 includes the following tasks:

1-1 Identify, catalog, and document existing one-dimensional (1D), two-dimensional (2D), and threedimensional (3D) nonlinear soil constitutive models appropriate for U.S. NPPs. Identify and develop new models, as needed, using a programming language suitable for inclusion in MOOSE (see http://www4vip.inl.gov/research/moose-applications/) and the commercial codes listed

\footnotetext{
$8 \quad$ https://earthquake.inl.gov/SitePages/Mastodon.aspx
}

9 SHAKE (Schnabel, Lysmer, and Seed 1991) and SASSI (Ostadan 20016a, 2006b) are legacy codes in the nuclear community and have been used for decades. As such, they have not been subject to formal verification and validation of the type described here. It is hoped that the utility (range of application) of these legacy codes will be assessed using the datasets generated in the experiments described here.

10 http://seismic-research.inl.gov/SitePages /Home.aspx. 
previously. Implement each model in MOOSE after verification and validation activities are completed.

1-2 Verify 1D, 2D, and 3D nonlinear constitutive models. Develop, solve, and document example problems. Follow the verification procedures set forth in ASME (2006) and Oberkampf and Roy (2010), and develop a procedure specific to soils suitable for others to follow later.

1-3 Plan and execute 1D, 2D, and 3D static and dynamic element-level tests on soil samples. Plan and execute cyclic, dynamic 2D and 3D simple shear tests at different confining pressures (similar to the Kammerer, Pestana, and Seed [2002] tests on undrained Monterey sand) to support validation of new and existing models for analysis at sites of nuclear facilities. Document, curate, and archive tests, test results, instrumentation, and metadata to allow validation of models by others.

1-4 Validate 1D, 2D, and 3D nonlinear constitutive models using experimental and field data. Validate constitutive models using test and field data. Follow the validation procedures set forth in ASME (2006) and Oberkampf and Roy (2010); develop a procedure specific to soils suitable for others to follow at a later time.

1-5 Verify accuracy of computer codes for SRA and SSI analysis. Develop trial problems for linear and nonlinear SRA and SSI analysis and solve using finite elements and constitutive models available in commercial and open source codes; investigate utility of alternate shape functions, procedures for discretizing the soil column, and required time step for implicit analysis; use simplified inputs such as wavelets (Ryan 1994, Bolisetti and Whittaker 2015) and simple soil columns that enable closed form solutions where possible. Follow the verification procedures set forth in ASME (2006) and Oberkampf and Roy (2010).

1-6 Plan and execute tests in a 1D geotechnical laminar box. Plan and execute a coordinated series of tests of alternate soil columns, with and without surface-founded and fully embedded structures, with dry and saturated soils, in a 1D geotechnical laminar box. Place only those soils that can be represented with verified and validated constitutive models in the box. Instrument the laminar box with transducers capable of measuring the response of the soil column and the inter- and intra-earthquake-shaking evolution of soil mechanical properties. Input low- and high-intensity ground motions to the base of the soil column to generate linear and highly nonlinear response of the soil columns, suitable for validation of both equivalent linear and nonlinear SRA and SSI numerical codes. Measure basemat and sidewall pressure histories. Document, curate, and archive tests, test results, instrumentation, and metadata to allow validation of models by others. Execute tests in an NQA-1 accredited laboratory, if possible.

1-7 Validate accuracy of computer codes for SRA and SSI analysis. Validate commercial computer codes using test data. Follow the validation procedures set forth in ASME (2006) and Oberkampf and Roy (2010); develop a procedure specific to soils and the 1D geotechnical laminar box suitable for others to follow at a later time.

1-8 Plan and execute gapping and sliding tests. Plan and execute a series of earthquake-simulator tests (http://seesl.buffalo.edu) of simple blocks of varying mass distributions and densities, installed atop a shallow soil deposit, with the explicit goal of triggering gapping and sliding. Input 1D, 2D, 3D, and 6-degree-of-freedom inputs of varying intensities. Instrument the soil and the blocks to monitor total and relative responses, bearing pressures beneath the blocks, and changes in material properties (soil only). Document, curate, and archive tests, test results, instrumentation, and metadata to allow validation of models by others. Execute tests in an NQA-1 accredited laboratory, if possible.

1-9 Validate accuracy of computer codes for gapping and sliding. Validate computer codes using test data. Follow the validation procedures set forth in ASME (2006) and Oberkampf and Roy (2010). 


\subsubsection{Assessment of Application and Limitations of 1-Dimentional Assumption in Site Response Analysis}

As discussed in Section 2.1.2 of INL/EXT-15-36945, SRA is a necessary precursor to SSI analysis, regardless of the type of analysis (e.g., linear, equivalent linear, nonlinear) or the number of components of earthquake input $(1,2$, or 3$)$. Traditionally, SRA has assumed 1D vertically-propagating shear waves as input to the structure. However, Section 4.2 of NRC Regulatory Guide (RG) 1.208 (NRC 2007b) states:

"Often vertically propagating shear waves are the dominant contributor to free-field ground motions at a site. In these cases, a one-dimensional equivalent-linear analysis or nonlinear analysis that assumes vertical propagation of shear waves may be appropriate. However, site characteristics (such as a dipping bedrock surface, topographic effects, or other impedance boundaries), regional characteristics (such as certain topographic effects), and source characteristics (such as nearby dipping seismic sources) may require that analyses are also able to account for inclined waves."

Although Section 4.2 of RG 1.208 raises important issues related to analysis of sites for which the traditional assumption of 1D vertically-propagating shear waves may not apply, regulatory guidance on appropriate tools and methods for 2D or 3D analysis is not currently available. This is an important gap for all structures - particularly the deeply-embedded structures for which the 1D assumption is more suspect. NLSSI provides an approach that could be directly applied in these instances to meet both SRA and SSI analysis needs.

An approach to address this issue could begin with using the new capabilities recently added to MASTODON, developed within the MOOSE framework, to understand whether and how waves other than $1 \mathrm{D}$ vertically propagating shear waves impact a variety of reactor structural configurations, with a focus on deeply-embedded advanced reactors. ${ }^{11}$ Once it is determined which waveforms significantly impact different NPP designs, the waveforms could be tied back to the sources that cause them (e.g., steeply dipping bedrock or soil layers, significant surface waves, etc.). This effort is tied directly to research on appropriate ground motion selection and modification (GMSM) techniques, as discussed in the next subsection.

Topic 2, which addresses a lack of guidance related to cases where the assumption of 1D vertically propagating waves may not apply, is considered a high priority, near- to medium-term effort. The following are subject areas that require technical research, development, and deployment. Topic 2 must be addressed to support the deployment of deeply-embedded advanced reactors. Work in these areas is needed to allow for an accurate estimate of seismic demands, which is needed for design and SPRA activities. To enable widespread and speedy dissemination, all research-oriented products would be made available via the INL Seismic Research Group website described previously.

Addressing Topic 2 includes the following tasks:

2-1 Develop stylized designs for example deeply-embedded advanced reactors. To determine the importance of wave fields that are different from the traditional 1D assumption, stylized designs are needed of stylized embedded reactors for a number of site conditions (soil types, layering, etc.) suitable for the construction of advanced reactors.

11 INL has recently implemented the Domain Reduction Method as originally developed by Bielak (e.g., Bielak et al. 2003 , Yoshimura et al. 2003), in MOOSE. Analysis using MOOSE for example sites and geological domains, and hypothetical fault rupture scenarios, would be used to propagate rupture-induced seismic waves to (1) the 3D boundary of a soil domain that will include the nuclear facilities, and in a subsequent analysis propagate the 3D wave field around the boundary of the soil domain into the facilities; and (2) the center of the lower boundary of the soil domain that will include the nuclear facilities, and in a subsequent analysis apply the three-component time series across the lower boundary of the soil domain and propagate the motions up into the nuclear facilities. Such analyses should be performed for a variety of deeply-embedded advanced reactor configurations to gain a clear understanding of the relative seismic demands imposed by inclined body waves and surface waves. 
2-2 Develop acceleration time series appropriate for input to SSI models. Acceleration time series for SSI analysis, consistent with a range of seismic wave types and wave forms that would result from a near-surface sloping underlying bedrock will need to be generated for SSI analysis of the stylized reactors of Task 2-1. This effort would incorporate and build on work now underway by expert earth scientists and engineering seismologists at the Southern California Earthquake Center (SCEC).

2-3 Determination of cases where the 1D assumption is insufficient. Using the DRM-based capabilities recently added to MOOSE, case studies need to be performed to understand the impact of inclined waves, wave passage effects, and possible shielding by adjacent structures on the seismic response of the example reactors of Task 2-1 for design and SPRA calculations. Results would be benchmarked against those calculated using traditional representations of ground motion input at the same lower boundary of the soil domain used for the more complex wave field. Once it is determined what wave forms are significant for which embedded reactors and sites, the wave forms can be tied back to the sources that cause them (e.g., steeply dipping bedrock or soil layers, significant surface waves).

2-4 Guidance development. Guidance on establishing the required definition of the seismic wave field (1D, 2D, or 3D) for design and SPRA need to be established using the stylized reactors of Task 2-1, the wave fields of Task 2-2, and the simulations of Task 2-3. Recommendations need to be integrated with the products of Topic 3 (see Section 2.1.3). The guidance needs to be prepared for possible adoption in the next editions of ASCE/SEI 4 and ASCE/SEI 43, and for consideration by the NRC.

\subsubsection{Ground Motion Selection, Modification and Horizon Definition}

GMSM has been a subject of much research for more than a decade, and there is no optimal solution for all applications. A report by the National Institute of Science and Technology (NIST 2011) provides a large body of information on this important topic. As discussed in INL/EXT-15-36945, Section 2.1.5, a regulatory guidance gap exists due to a lack of definitive guidance on the GMSM of ground motions generally for all SRA and SSI analysis. However, because legacy approaches exist for traditional surfacefounded NPPs, the gap is most problematic for facilities with nonlinear soil-structure systems, which includes all NPPs with SI and many advanced reactors.

Currently, the state-of-art in SSI analysis utilizes three-component ground motion records applied to the lower boundary of a soil domain (i.e., a rock or hard soil horizon). These three-component motions are developed using hazard-consistent spectra determined by probabilistic seismic hazard analysis (PSHA) (see NRC 2007b and NRC 2012). The ground motion inputs used in SSI analysis must be consistent with any SRA performed as part of the PSHA, particularly if the SRA is directly implemented in the ground motion characterization model. While this configuration with input at a single layer may be appropriate for some surface- or near-surface-founded reactors (depending on the geologic profile at the site), there will be additional considerations for deeply-embedded reactors or reactors with height to width aspect ratios different from LWRs. These considerations include wave incoherency and the relative amplitudes of input motions at the various soil-structure interfaces. The approaches most appropriate for advanced reactors, deeply-embedded reactors, NPPs with height to width aspect ratios different from LWRs, and reactors with risk-significant frequency ranges differ from those of LWRs. As a result, the identified gap related to GMSM is most acute for advanced reactors.

As discussed in INL/EXT-15-36945, Section 2.1.5, at least four procedures exist for generating threecomponent acceleration time series currently used in the technical community. The procedure that matches horizontal motions to the geomean horizontal uniform hazard response spectrum from the PSHA with explicit recognition of the difference in amplitude of the two horizontal components (Huang, Whittaker, and Luco 2008a, 2009a; Huang et al. 2009b) is considered the most robust, especially for nonlinear response-history analysis (Huang, Whittaker, and Luco 2008a). Kumar et al. (2015b) performed 
analysis on nonlinear isolation systems (fundamentally no different from a nonlinear soil-structure system) and concluded that this procedure was best for generating mean and 90th percentile demand values in an SSI analysis.

Topic 3, which addresses a lack of guidance related to ground motion selection and modification for SI and NLSSI, is considered a medium-priority, medium-term effort. The following are subject areas requiring technical research, development, and deployment. All three are topics that should be addressed to support the deployment of deeply-embedded advanced reactors. Work in these areas is needed to allow for accurate estimate of seismic demand, which is needed for design and SPRA activities. To enable widespread and speedy dissemination, all research-oriented products would be made available via the INL Seismic Research Group website described previously.

Addressing Topic 3 includes the following tasks:

3-1 Spectral representation and scaling. Although the Huang procedure discussed above is likely to yield the most realistic results in terms of soil shear strains, isolator displacements and demands on SSCs, this has not been demonstrated. Analysis of example stylized soil-structure systems, intended to represent a range of surface-mounted and deeply-embedded advanced reactors, should be conducted for ground motions developed using a range of currently used GMSM procedures for design and seismic performance assessment. Recommendations will be developed for linear and nonlinear SRA and SSI.

3-2 Minimum number of sets of ground motions. Determine the minimum number of sets of threecomponent ground motions required to (1) compute design basis demands at the 80th percentile, (2) calculate beyond design basis demands on isolators at the 90th percentile, and (3) perform the SPRA for deeply-embedded advanced reactors, with considerations of isolated components and systems. Example stylized deeply-embedded advanced reactors, equipped with isolated components, need to be proposed and analyzed for this purpose.

3-3 Guidance development. Recommendations for selection and scaling of sets of ground motions, including methods and numbers of sets, will be needed for linear and nonlinear SRA and SSI analysis, for both design and SPRA of deeply-embedded advanced reactors. The guidance needs to be prepared for possible adoption in the next editions of ASCE/SEI 4 and ASCE/SEI 43.

\subsection{Isolation of Equipment: Tools and Guidance}

The physical scale of equipment in nuclear facilities spans from kilograms to thousands of tons and from the size of a shoebox to the size of a reactor vessel. The shapes of equipment also vary widely. Isolators and isolation systems developed for base isolation of nuclear facilities weighing thousands to hundreds of thousands of tons may not be appropriate for equipment housed in conventionally founded structures. For example, a four-isolator system for a cabinet in a deeply-embedded advanced reactor will be fundamentally different from a foundation isolation system for a large LWR, which could be composed of hundreds of isolators. The cabinet may need isolation system configurations utilizing existing 2D isolators, prestressed 2D isolators, or new types of isolators (including those capable of 3D protection); whereas, traditional 2D isolators will likely suffice for the base isolation case.

Isolation systems for equipment (likely to be found at the low-end to mid-range of the size, geometry and weight distributions considered here), have yet to be developed, prototyped, and deployed, although advances have been made in the past few years. A family of isolator types and isolation-system solutions suitable for use by the nuclear community should be developed to meet the need. Supplemental energy dissipation devices, including fluid viscous dampers and metallic yielding devices (e.g., ADAS elements, buckling restrained braces), may augment or be key components of an isolation system for a piece of equipment. 
Existing regulatory guidance (e.g., draft NUREG/CR) and standards (e.g., ASCE 4-16, Chapter 12) address the analysis and design of base-isolated nuclear facilities assuming the entire nuclear structure is protected by a plane of isolation bearings. There is no guidance or standards language specific to equipment, which experiences seismic input from the structure through its support or anchorage system. There is no guidance related to umbilical lines that would cross the interface between the isolated piece of equipment and surrounding, non-isolated SSCs. Further, there is no guidance or standards language for equipment that is isolated horizontally and/or vertically at one level and restrained horizontally at other levels (e.g., a steam generator). New guidance on analysis and design of equipment isolation systems and testing of the isolators (and possibly small systems) would significantly benefit deployment in advanced reactors (deeply embedded or not), new large LWRs, and possibly in the existing fleet of LWRs.

Topic 4, which addresses a lack of tools and guidance related to isolation of equipment, is considered a high-priority, medium-term effort. The following R\&D activities are needed to support commercial deployment of equipment-oriented SI technologies at advanced reactors. To enable widespread and speedy dissemination, all research-oriented products would be made available via the INL Seismic Research Group website identified previously.

4-1 Identify candidate components of advanced reactors for seismic protection. Engage with designers of advanced reactors to identify components of advanced reactors that are candidates for seismic protection using isolators and supplemental damping devices. Bin the identified components by physical size, mass, and geometry to enable development of example stylized generic equipment isolation and damping systems.

4-2 Develop example non-proprietary seismic protective solutions for candidate components. Develop seismic protective solutions (isolators and damping devices) for the binned components of Task 4-1, accounting for possible installations in high radiation environments. Estimate instructure seismic demands for design of the protective solutions by analysis of an example stylized deeply-embedded advanced reactor, equipped with the components of Task 4-1, for shaking consistent with design basis and BDB at sites of existing NPPs in the U.S. Develop numerical models of the seismic protective solutions and verify per ASME best practice (ASME 2006).

4-3 Validate numerical models for the seismic protective solutions. Execute large-scale 3D earthquake simulator experiments on the seismic protective systems of Task 4-2. These tests would create datasets to validate the numerical models of the seismic protective solutions per ASME best practice (ASME 2006). Document, curate, and archive test configuration and procedure information, test results, information on instrumentation, and other test metadata to allow validation of models by others. Execute tests in an NQA-1 accredited laboratory, if possible.

4-4 Identify performance criteria for protected components consistent with NRC goals. Risk-informed performance-based criteria form the basis of the mandatory language of ASCE/SEI 4-16, Chapter 12, and the guidance of the draft NUREG/CR, for DOE facilities and U.S. NPPs, respectively. These criteria meet the risk targets of DOE and NRC, respectively. Similar criteria must be identified for seismically protected equipment, consistent with the proposed Seismic Category ${ }^{12}$ for the equipment. (The choice of Seismic Category may dictate the risk target for the component and thus the definition [mean annual frequency of exceedance] of beyond design basis shaking.) Because 3D isolation of equipment will be possible, the performance criteria of ASCE/SEI 4-16 and the draft NUREG/CR need to be extended to address vertical isolation.

4-5 Develop guidance for analysis, design, and testing of seismically protected equipment. Guidance for the analysis and design of equipment seismically protected by isolation systems in 2D (horizontal plane) and 3D (including vertical shaking) will be needed to support the design and

\footnotetext{
${ }^{12}$ Seismic Categories are discussed in NRC Regulatory Guide 1.29 (NRC 2007c).
} 
licensing of reactors using this technology. Guidance will need to address the use of innovative isolators and isolation systems, the possible use of supplemental damping (energy dissipation) devices, seismic input at multiple attachment points (vertical and horizontal) of the equipment anchorage in the NPP, construction and operational considerations, and Seismic Category. Procedures for dynamic testing of components of the seismic protective systems and appropriate acceptance criteria for both prototype and production testing programs, consistent with the chosen performance goal(s), will need to be written. Scale model testing of the protective systems of Task 4-2, consistent with the mandatory language of ASCE/SEI 4-16, Section 12.1, for new 2D seismic isolation systems, will likely be required to support the guidance. These 3D earthquake-simulator tests should be performed in an NQA-1 accredited laboratory, if possible. The guidance should also address approaches for seismic qualification of isolation system components and minimum FIRS for isolated equipment. The guidance should be appropriate for possible adoption in Chapter 12 of the next edition of ASCE/SEI 4, likely to be published in 2021. The guidance should also be appropriate for NRC review.

\subsection{Design and Analysis Considerations for the Stop}

As discussed in more detail INL/EXT-15-36945, Section 2.2, the need for a physical displacement restraint, or stop, is referenced in both the draft SI NUREG/CR report and the SI provisions of ASCE/SEI 4-16, and performance criteria are provided in these documents. The stop is intended to limit displacement of an isolated superstructure and to limit the deformations in the isolator units and any crossover umbilical lines.

The guidance provided in the draft SI NUREG/CR report and the mandatory language of ASCE/SEI 4-16 are applicable to a wide range of superstructures and equipment, ranging from an emergency diesel generator supported by four isolators to a nuclear island supported by hundreds of isolators. The appropriate design details of the stops for these isolated SSCs, which range in weight from tens of tons to hundreds of thousands of tons, will be vastly different. This is one reason that neither the draft SI NUREG/CR report nor ASCE/SEI 4-16 provide detailed guidance on the design of a stop.

An impediment to the implementation of SI in nuclear facilities is the lack of analysis, design, and detailing examples for stops, especially for isolated components supported in advanced reactors, whether deeply embedded or near-surface mounted. Examples that span a range of isolated components in nuclear structures (e.g., emergency diesel generators, cabinets) have not been prepared, but would be useful. Although the draft SI NUREG/CR report offers a procedure to identify an impact velocity for an isolated nuclear facility, no guidance is available for the velocity calculation for an isolated component in an advanced reactor.

Topic 5 addresses the analysis and design of stops for equipment isolated inside advanced reactors, which is considered a high priority, medium-term effort. Analysis and design procedures for a stop for a base-isolated facility, is a lower priority, longer term effort and so not addressed here. The following SI technology R\&D activities are needed to enable deployment of SI for components (or equipment) in an advanced reactor. To enable widespread and speedy dissemination, all research-oriented products would be made available via the INL Seismic Research Group website identified previously.

Addressing Topic 5 includes the following tasks, building on the outcomes of Topic 4:

5-1 Develop trial stylized designs for stops for example isolated components. Analyze and design stylized 2D and 3D stops for the isolated components (or equipment) in the advanced reactor of Topic 4, considering a range of NPP sites. Document the procedures used and construction details to speed adoption by the nuclear community. Develop simplified analysis and design procedures for 2D and 3D stops, and document. Validate 2D and 3D stop construction details by scale model testing. 
5-2 Develop guidance for analysis, design, and testing of stops for seismically protected equipment. Guidance for the analysis and design of 2D and 3D stops for seismically protected equipment in 2D (horizontal plane) and 3D (including vertical shaking) will be needed to enable deployment in the field. Guidance will need to address the innovative isolators and isolation systems of Topic 4, seismic input at multiple attachment points (vertical and horizontal) of the equipment in the NPP, and Seismic Category. The guidance developed should be suitable for consideration by NRC and the Dynamic Analysis of Nuclear Structures committee of ASCE/SEI Standards 4 and 43. The guidance will be prepared for possible adoption in next editions of ASCE/SEI 4 and ASCE/SEI 43.

\subsection{Seismic Probabilistic Risk Assessment}

As discussed in more detail in Section 2.3 of INL/EXT-15-36945, SPRA is used for a range of regulatory purposes in the U.S., and its use is increasing globally. In addition to the regulatory uses, the role of SPRA in informing design activities also continues to evolve. The benefits of SI will be quantified in the SPRA in the form of lower seismic motions in the superstructure, which translate into much smaller demands on isolated SSCs. Although SI has been shown to reduce facility-level risk, when SPRA is performed for an NPP using SI technology, additional failures may need to be considered and assessed and incorporated into the accident sequences of the plant response model, as appropriate. These include failure of the isolation system, failure of umbilical lines and other crossover SSCs, and the potential for pounding against the stop.

INL/EXT-15-36945, Section 2.3, identified regulatory challenges and gaps associated with performing an SPRA for an isolated surface- or near-surface-founded reactor, a deeply-embedded reactor, and an NPP in which components and systems are isolated. The following sections identify approaches for addressing gaps and challenges related to SPRAs performed for NPPs using SI technology. It is important to note that risk metrics for some advanced reactors may be different from those for LWRs, for example, core damage frequency may not be relevant. Below we emphasize unacceptable performance rather than core damage frequency (for example) to be technology-neutral.

\subsubsection{Seismic Probabilistic Risk Assessment Calculations for Failure of the Isolation System and Umbilical Lines}

Traditional SPRA methods that employ a standard ground motion spectral shape anchored to peak ground acceleration (PGA) cannot be used for nuclear structures isolated with nonlinear seismic isolation bearings because in-structure response spectral demands do not scale proportionally with shaking amplitude. Nonlinear methods of analysis are needed to predict responses in isolated nuclear structures, and the interaction of nonlinear soils and isolators will generally require fully coupled NLSSI analysis.

Accident sequence initiators to be considered for a seismically-isolated NPP are (1) failure of the isolation system, wherein the isolation system is unable to sustain axial loads imposed by gravity and earthquake shaking at the horizontal (lateral) displacements expected in BDB shaking, and (2) failure of the safety-related umbilical lines that cross the isolation interface (i.e., from isolated construction to non-isolated construction). As described above, a physical stop is introduced in ASCE/SEI 4-16 and the draft SI NUREG/CR report to limit the horizontal displacement of an isolated superstructure to well below its capacity. The requirements for implementing a stop and dynamic testing of prototype isolators to the lateral displacement defined by the stop are intended to ensure the mean annual frequency of failure associated with isolation-system failure is less than $10^{-6}$ if the recommended NRC performance criteria are used. The stop also limits the deformation in the umbilical and crossovers.

Examples of risk-based calculations for assessing adequacy of an SI system design and demonstration of the risk-benefits of seismically-isolated surface or near-surface-founded LWRs are provided in Huang, Whittaker, and Luco (2008b, 2011a, 2011b); Kumar, Whittaker, and Constantinou (2015b); and Kumar and Whittaker (2016). The Huang, Whittaker, and Luco report and papers provide a methodology for the seismic performance assessment (risk calculations) of an isolated NPP, as presented in 
INL/EXT-15-36945, Section 2.3.1. In the example analysis, the NPP is equipped with linear (low damping rubber) and nonlinear (lead rubber, friction pendulum [FP]) bearings. These calculations assume a rigid surface beneath the isolation systems to address compliant nonlinear soil and deeply-embedded reactors. Huang, Whittaker, and Luco (2008b, 2011b) quantify the benefits of implementing SI for stylized, simple accident sequences involving failure of the isolation system. An extensive discussion of the methodology used is provided in INL/EXT-15-36945 and is not repeated here. The peer-reviewed methodology is also referenced in ASCE/SEI 4-16.

Kumar, Whittaker, and Constantinou (2015b) and Kumar and Whittaker (2016) provide a risk-calculation procedure for SI systems and safety-related umbilical lines that cross the isolation interface. As noted above, the possible deformations of the SI system and umbilical lines are limited by the stop.

The above discussion and methods developed focuses principally on surface- or near-surface foundations. A deeply-embedded reactor will experience seismic loads along its basemats and walls. These loads cannot be characterized by a surface free field acceleration response spectrum for analysis purposes. SRA and SSI analysis for design and SPRA for such reactors will need to employ an alternate horizon control point (or control points) to define seismic input. The horizon to be used for analysis will likely be located at some depth below the reactor.

The Huang, Whittaker, and Luco methodology described above was developed for SPRA of isolated surface-founded LWRs. However, this methodology can be applied for risk calculations for deeply-embedded advanced reactors with some modifications. In this case, soil above the rock horizon and surrounding the embedded reactor is modeled explicitly (replacing the nonlinear isolators) and the three-component ground motions are applied at the rock horizon below the base of the embedded reactor (replacing the motions applied at the underside of the isolation system). The calculations would proceed identically to those used for surface-founded NPPs, except that the ground motion and inputs are appropriate for deeply-embedded reactors. Research and development activities are needed to support commercial deployment of deeply-embedded advanced reactors, regardless of whether they utilize SI technologies.

Topic 6, which addresses needs related to incorporating SI into SPRA, is a high priority, short- to medium-term effort. Topic 7, which develops a demonstration of SPRA for an isolated NPP, is a medium priority, medium-term effort. The following technology R\&D activities are needed prior to commercial deployment of SI in advanced reactors. To enable widespread and speedy dissemination, all researchoriented products would be made available via the INL Seismic Research Group website identified previously.

Addressing Topic 6 includes the following tasks:

6-1 An advanced SPRA-based methodology is required to assess the risk of failure of the foundation system in seismically-isolated nuclear facilities. The Huang, Whittaker, and Luco (2008b, 2011a, 2011b) SPRA-based methodology needs to be implemented in MOOSE to enable risk assessment of isolated nuclear structures.

6-2 Develop process for assessing fragility of umbilical lines using both the Huang et al. SPRA methodology and using traditional SPRA.

6-3 Characterize the assumption of isolator correlations. The fragility functions of Kumar, Whittaker, and Constantinou (2015b) and Kumar and Whittaker (2016) are biased low because the failure of one isolator is assumed to represent failure of the isolation system. This is a very conservative assumption that assumes that the isolator axial force and horizontal displacement demands are fully correlated, which will almost never be the case. The impact of this assumption on risk calculations needs to be quantified for example applications. 
6-4 Develop a process for determining demands to SSCs for a traditional SPRA.

Addressing Topic 7 includes the following tasks:

7-1 Develop example stylized designs of civil/structural systems and safety-related SSCs (including fragility functions) for an isolated advanced reactor(s) to use in a example SPRA.

7-2 Develop accident sequences (event trees and fault trees) for stylized reactor designs. Event trees and fault trees will be needed to develop systems models for the stylized reactor and enable SPRA.

7-3 Perform SPRA calculations for stylized reactors and develop documents with guidance and examples.

\subsubsection{Seismic Probabilistic Risk Assessment Calculations for Isolated Components}

It may be unlikely that deeply-embedded reactor buildings will be seismically (base) isolated for two reasons. First, there are significant additional initial construction costs associated with the over-excavation of the site. The additional excavation is required below the basemat to enable placement of the foundation, pedestals, and isolators and around the building to provide uninterrupted space for the isolated building to displace. Secondly, there are additional costs associated with construction, monitoring and maintenance of a permanent retaining wall surrounding the embedded building. These additional costs will likely outweigh the benefits in terms of reduced seismic inputs. However, deeply-embedded reactors may benefit significantly from the isolation of safety-related components such as reactor vessels, as measured by increased seismic safety and/or reduced cost. Figure 2 presents cut-away views of examples of applications of seismic protective systems, defined here as a combination of SI devices and energy dissipation devices, such as fluid viscous dampers.

The need for guidance related to design of isolation of equipment and systems is found in Section 2.2 of this report. Considerations related to the licensing of NPPs with equipment isolation within the certified design framework is found in Section 3.1. There is also a need for the development of guidance on incorporating isolated equipment into SPRA.

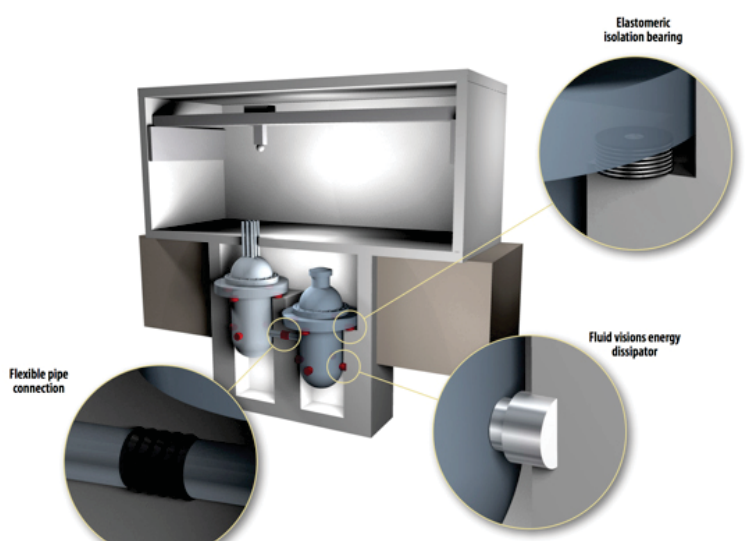

a. Component isolation
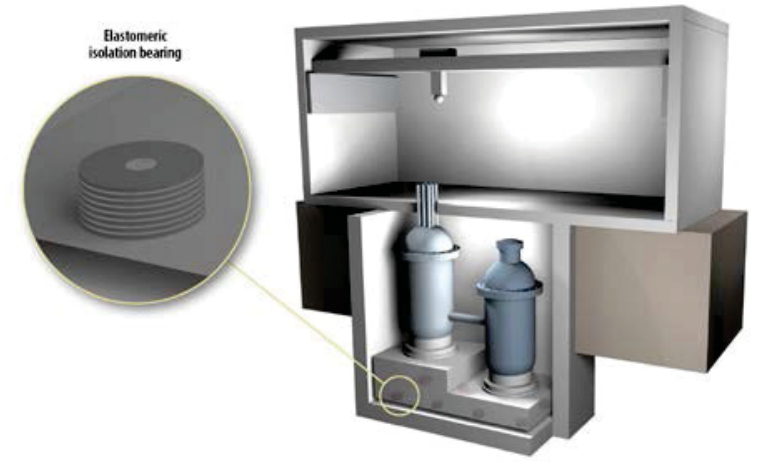

b. Internal system isolation

Figure 2. Deeply-embedded advanced reactor incorporating seismic protective systems.

Topic 8, SPRA approaches for isolated components, is a high priority, medium-term effort. The following are technology R\&D activities that should be conducted to enable deployment of isolated equipment. The activities listed below are predicated on the prior completion of the several items in Task 4 in Section 2.2.

Addressing Topic 8 includes the following activities: 
8-1 Develop example stylized designs of protective systems for components. Selected safety-related SSCs would need to be identified for possible isolation. Seismic design spaces would need to be developed for two cases for each SSC. In the first case, the SSC capacity would be based on non-seismic load cases. In the second case, the capacity would be based on the stylized designs of Task 4. Protective systems (isolators and dampers) for each case, site and soil profile would be developed. For each design, the cost needs to be developed to establish the benefit, if any, of the use of protective systems.

8-2 Develop fragility methods for the protective systems and safety-related umbilical lines. Develop example event trees and fault trees needed to develop the plant response analysis based on stylized designs developed in Task 4.

8-3 Perform SPRA calculations for the stylized reactors. The analysis, design, and risk assessment of the example stylized reactors (one reactor type, multiple sites, multiple soil profiles), coupled with the careful documentation thereof, would provide a methodology that could be used by analysts, designers, and regulators. Importantly, the SPRA calculations would inform and guide the possible use of protective systems for large equipment and systems.

8-4 Development of guidance for incorporating isolation systems for large systems and components into SPRA.

\subsubsection{Human Reliability in Isolated Facilities}

The approaches currently applied for quantification of reliability of operator action in SPRA have been in use for decades and, until recently, few advances have been developed and proposed for use. Generally, the probability of failure of a manual action in a seismic event is treated as a set multiplier (e.g., multiplication by 2) of the expected probability of failure for a non-seismic event, which itself has a high degree of uncertainty. The probability of failure includes an assumption that some portion of the NPP operations staff is unable to perform their function due to a blocked access path, injury, or some other factor. Although seismic walkdowns often look for the potential for important pathways to be blocked as a result of physical damage to SSCs during a seismic event, a systematic treatment that includes a thorough investigation of all pathways that may be needed is not universally applied. This is despite the current understanding that some accident sequences that are determined to be risk-significant in SPRAs may have some element of operator action. Given this current state of practice, the benefit of SI in terms of risk reduction resulting from improved human performance would not be fully incorporated in the SPRA model and results. Also potential adverse impacts, if any, have not been investigated and assessed.

Recently, work by EPRI (Presley et al. 2013a, 2013b) has identified a new approach that bases the probability of failure of operator action on plant damage state. This approach, though in the early stages of development and implementation, provides an opportunity to include a more technically-advanced approach to quantify risk in both isolated and non-isolated facilities. During reviews, the Seismic Advisory Group noted the need not to duplicate the EPRI work and recommended to wait on further human-reliability actions until the EPRI work has further matured.

As a result of input by the INL Seismic Advisory Group, Topic 9, which focuses on human factors in isolated NPPs was assigned a low priority. Addressing Topic 9 is limited to Task 9-1, which involves following the work of EPRI as it progresses.

9-1 Follow the work of EPRI on human factors and incorporate into the SPRA for SI designs, as appropriate.

\subsubsection{Guidance for Seismic Probabilistic Risk Assessment Feedback to Design}

SPRA performed as part of new reactor licensing activities has most commonly been performed towards the end of the design process. Although current performance and risk objectives for new NPPs 
designs are set facility-wide, they are most typically applied on an SSC by SSC basis, which leads to inefficiencies in NPP design (Budnitz and Mieler 2016). As discussed in INL/EXT-15-36945,

Section 2.3.5, infusing insights from risk calculations into the various stages of a design process has a number of important benefits. INL/EXT-15-36945 discussed how SPRA activities and insights could be integrated into the design process as it proceeds, from conceptual design through construction documentation.

Importantly, recent experience has shown that an integrated design-SPRA process will facilitate determining whether SI is economically beneficial for new advanced reactor designs. This process would optimize design in the early stages in order to support both economic and licensing efforts by making risk targets an integral aspect of design, along with the more traditional margins approaches. However, the Seismic Advisory Group noted that this work would likely be more effectively performed after more experience is gained in design of new NPPs using seismic isolation.

Topic 10, developing guidance for integrating SPRA insights into design, was identified as a medium priority, medium-term effort. The following technology R\&D activities would support commercial deployment of SI technologies at advanced reactors. To enable widespread and speedy dissemination, all research-oriented products would be made available via the INL Seismic Research Group website identified previously.

10-1 Develop guidance for design process that incorporates risk analysis as a fundamental element of the design process. This guidance should be based on recent and ongoing experience working with vendors on design.

\subsubsection{Generic HCLPF Capacity Tables for Isolated Nuclear Power Plants}

The incorporation of currently available SI technology reduces the response of a structure to horizontal ground motion through the installation of horizontally flexible and vertically stiff seismic isolators between the superstructure and its foundation. Whereas the horizontal accelerations are significantly reduced by the isolation layer, the vertical motions are relatively unchanged.

This outcome leads to a question of the applicability of commonly used generic high-confidence of a low-capacity of failure (HCLPF) capacity tables, such as those in Electric Power Research Institute (EPRI) Report NP-6041-SLR1 (EPRI 1991). The tables list equipment that has been shown to be robust to the ground motion level represented by the HCLPF capacity value during large seismic events in which the equipment was in service in industrial facilities. Because the equipment in the current database was universally housed in non-isolated facilities, the vertical to horizontal $(\mathrm{V} / \mathrm{H})$ ground motion ratios (commonly called the $\mathrm{V} / \mathrm{H}$ ratio) the equipment experienced would be different from those that would occur in an isolated facility. Therefore, as a result of the significant reduction of horizontal motion in an isolated facility, the $\mathrm{V} / \mathrm{H}$ ratio will be much higher than for the ground motions represented in the earthquake experience database.

Although it is believed that few SSCs are sensitive to vertical motions (with batteries being one common exception), it was a question as to whether it was necessary to address the use of the screening tables in SPRA for isolated reactors before the SPRA results are used for design or regulatory purposes. This question was posed to the Seismic Advisory Group, the members of which felt that the tables still applied. It was noted that the anchorage systems for the components, which are often the element with the lowest capacity, would be analyzed using the in-structure motions from SSI that account for the vertical load. Additionally, the evaluations should be conducted by analysts with appropriate experience and knowledge who can account for the differences in vertical loads. This may be addressed by ongoing efforts by EPRI to update and consolidate past fragility reports.

Topic 11, review of the generic HCLPF capacity tables to determine if they remain appropriate for use in isolated NPPs, was determined to be a low-priority, medium-term effort. The following technology R\&D activities may help to support commercial deployment of SI technologies at advanced reactors, but 
should not be given higher priority than other topics and activities. To enable widespread and speedy dissemination, all research-oriented products would be made available via the INL Seismic Research Group website identified previously.

11-1 Develop write-up of the history of the tables and discuss the potential issues related to vertical motion. This write-up should be included in one of the other related reports, rather than being published as a separate document. 


\section{CHALLENGES AND GAPS RELATED TO REGULATORY APPROACHES AND TERMINOLOGY}

\subsection{Terminology and Approach for Licensing Isolated Nuclear Power Plants Using the Certified Design Process}

As described in INL/EXT-15-36945, Section 3, the current approach for comparing the assessed ground motions at a site (based on PSHA results) against the ground motions used for a certified design is presented in DC/COL-ISG-017 (NRC 2009). This Interim Staff Guidance (ISG) further references NEI (2009) for additional details related to three possible approaches that can be applied, the choice of which depends on the NPP configuration. The possible configurations described reflect the LWRs going through licensing activities at the time of publication. Regardless of the approach utilized, the evaluation fundamentally compares the foundation input response spectra (FIRS), which are based on the sitespecific GMRS, to the CSDRS, as shown in Figure 3, which is a modified version of Figure 3-1 in NEI (2009).

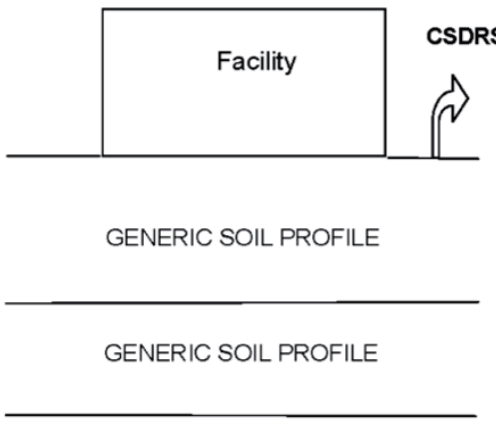

(a) SSI Model with Site-

Independent Generic Soil Profile
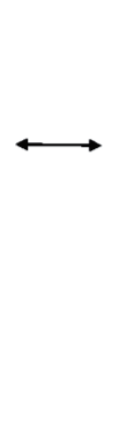

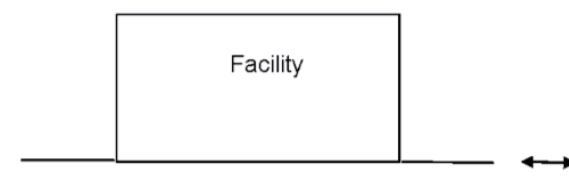

SOIL

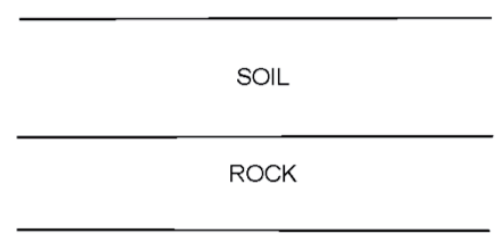

(b) Facility at the Site on Site Soil Profile

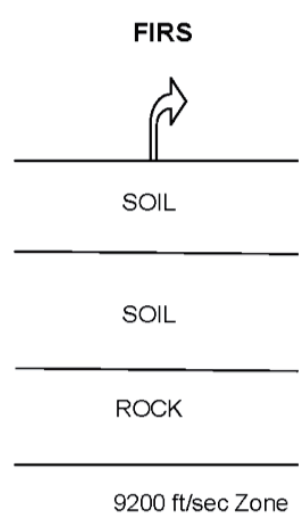

(c) Site Soil Profile used with Free-Field FIRS

Figure 3. Approaches for comparing site-independent certified seismic design response spectrum against site-dependent foundation input response spectrum (modified for clarity from Figure 3-1 of NEI [2009]).

Although the above approach comparing the FIRS to the CSDRS has been successfully applied for conventionally-founded LWRs, it cannot be directly applied to facilities with even simple base isolation configurations without careful consideration and clarification/modification because in a base-isolated structure the CSDRS and FIRS are not unambiguously co-located at a single control point. In current practice, the isolation system is designed on a site-specific basis that depends on the site-specific hazard and the dynamic properties of both the site and the isolated super structure (including any systems and components that may contribute to the structural response). ${ }^{13}$ Site-specific design optimizes the performance of the isolation system; however, this means that the isolation system would not be part of the certified portion of a facility and, as a result, the CSDRS location (which is site-independent and defined at the base of the certified portion of the facility) is separated from the soil/foundation interface, which is where the site-specific motions are traditionally assessed. If a site-specific isolated systems is developed, a new approach wherein the site-specific motions are compared to the certified design

13 Although the seismic isolation is site-specific in current engineering practice, this topic also looks at isolators being part of the certified design. Although this loses some of the benefits of seismic isolation and may prove problematic from a design perspective, it makes licensing under the certified design framework simpler in some ways. 
response spectrum at the top of the foundation (as shown by the red line in Figure 4), must be considered. The site-specific motions used for comparison to the certified design must account for the site-specific hazard, as well as, the isolation system's dynamic response to the expected ground motions. This represents a significant gap in the application of the certified design process to isolated NPPs generally.

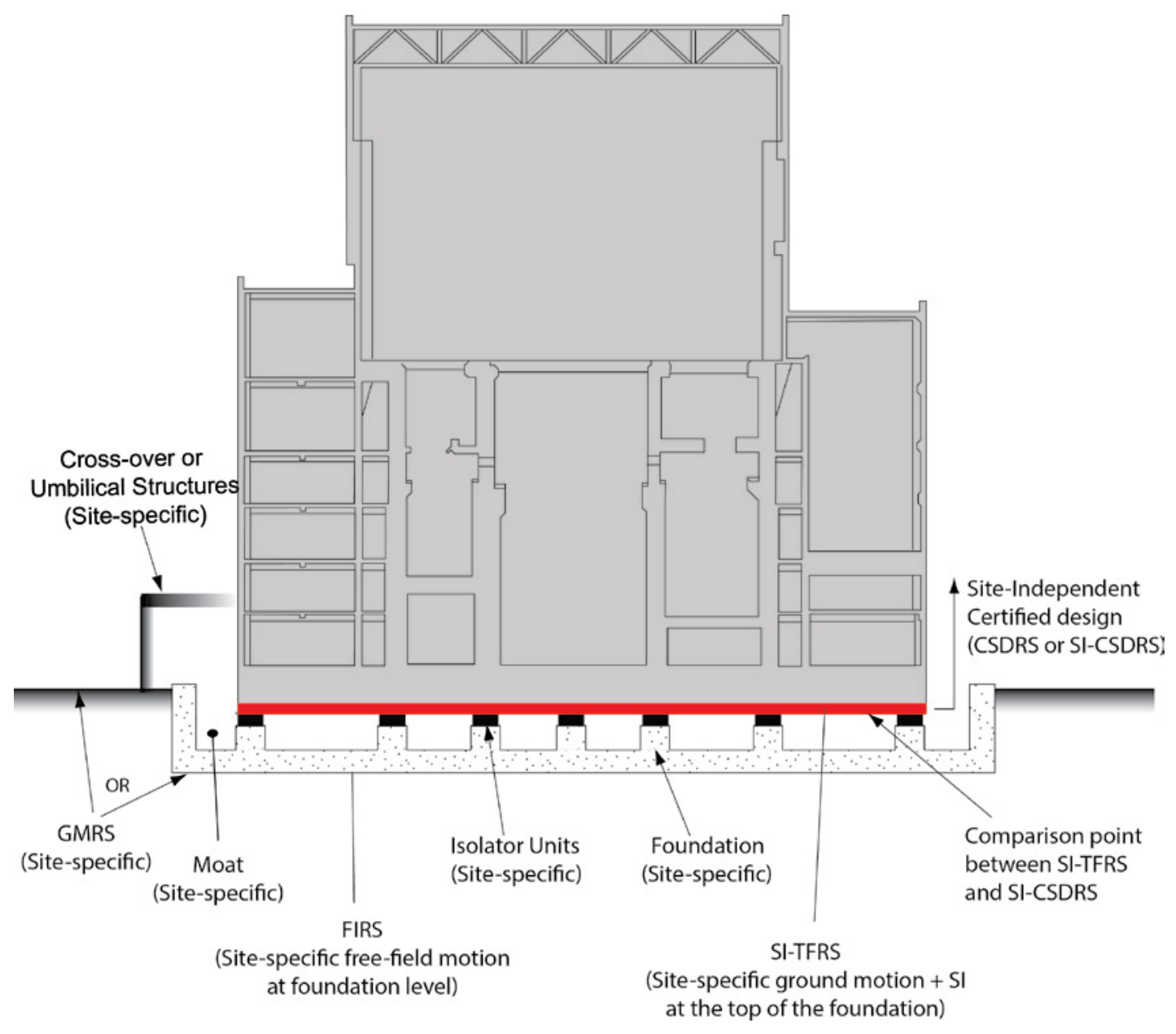

Figure 4. Diagram showing potential comparison horizon for evaluation of site against certified design for surface-founded, base-isolated nuclear power plant.

A modified regulatory approach, such as that described in INL/EXT-15-36945, Section 3, should be developed to close the gap in the certified design process related to SI. This modified approach will require new terminology to bring clarity to the design, review, and licensing process. Figure 4 identifies two possible new definitions proposed for use in the licensing of surface-founded base-isolated structures using the certified design approach. These proposed definitions are seismically-isolated top of foundation response spectrum (SI-TFRS) and seismically-isolated certified design response spectrum (SI-CSDRS) and are defined in Table 1. SI-TFRS and SI-CSDRS are both defined at the level of the upper basemat and provide the appropriate point for comparison, as shown in Figure 4. The terminology can be used to distinguish between licenses or license applications where a certified design can also be conventionally founded. 
Table 1. Definitions of proposed terms for use with seismically-isolated certified designs.

\begin{tabular}{|l|l|}
\hline \multicolumn{1}{|c|}{ Term } & \multicolumn{1}{c|}{ Definition } \\
\hline SI-TFRS & $\begin{array}{l}\text { Seismically-isolated top-of-foundation response spectrum. A site-specific motion defined at the } \\
\text { underside of the basemat and immediately above the plane of isolation in a base-isolated facility. }\end{array}$ \\
\hline SI-CSDRS & $\begin{array}{l}\text { Seismically-isolated certified design response spectrum. A site-independent motion in a } \\
\text { base-isolated facility, consistent with Subpart B of 10 CFR 52 and defined at the underside of the } \\
\text { basemat and immediately above the isolation system. }\end{array}$ \\
\hline
\end{tabular}

In addition to the above, license applications would be expected to provide analysis from a number example sites to demonstrate that the NPP could be sited in a range of locations. In this case, the analyses would require information on both the dynamic properties of the example site and appropriate SI system design for each example site. This would require input from SI vendors. An approach for developing and analyzing the example sites could be developed as part of this effort. Analyzing the example sites may provide insights into challenges and approaches related to vertical loads.

Because this issue is newly identified, and the consideration of approaches to address it are in the very early stages, a near-term effort is needed to further develop the concepts and appropriate supporting documentation to the NRC. This issue was originally identified during application of SI to a LWR, and so the potential path forward described above may only be applicable to surface-founded or near-surface-founded, base-isolated NPPs with isolators installed in a horizontal plane. Consideration must be given to additional situations. The range of cases to be considered include:

- Isolated facilities with foundation isolation at one level

- Isolated facilities with foundation isolation at multiple levels

- Isolation of existing certified designs

- Standard isolators included in certified design

- Isolation of systems and equipment

- Vertical isolation.

Many advanced reactor designs are deeply embedded with basemats at multiple levels. Although isolation of the structure surrounding the reactor vessel is unlikely for deeply-embedded designs for the reasons given in Subsection 2.4.2, it is possible. In such a case, and for a structure with basemats at multiple levels, neither a single SI-TFRS nor a single SI-CSDRS may provide the clearest and most optimal solution. That is because isolators at different levels will experience inputs that will be site-specific depending on local characteristics, including the dynamic characteristics of the soil below and surrounding the NPP. If a component within an advanced reactor is isolated (see Figure 2), it may experience out-of-phase seismic inputs at multiple levels. Alternate approaches to SI-TFRS and SI-CSDRS may be beneficial in these cases.

In addition to the questions of terminology and comparison locations, consideration should be given to the issue of how the vendor determines the standardized portion of the NPP. The separation between vendor and applicant responsibilities also needs to be clarified. Because the SI is site-specific, the burden would shift to the applicant unless it is explicitly shifted back to the vendor. The vendor is better equipped to address the licensing of the isolation system.

Additionally, Chapter 12 of ASCE/SEI 4-16 proposes SI design response spectra (SIDRS) for use with a multi-step process to account for the effects of SSI. The SIDRS are site-specific, are generated at the underside of foundation, and include the effects of SSI. Accordingly, SIDRS are different from both SI-FIRS and SI-CSDRS and this should be further clarified to avoid confusion. 
Topic 12, clarification of the certified design process for isolated reactors, was determined to be a high priority, short-term effort. The following are two tasks requiring a combination of study and development. These activities would reduce regulatory risk to the timely and efficient licensing of advanced reactors using SI technologies. To enable widespread and speedy dissemination, all research-oriented products would be made available via the INL Seismic Research Group website identified previously.

12-1 Evaluate best approaches using case studies and develop new terminology related to licensing of advanced reactors using the certified design process. This needs to address base isolation, multilevel foundations, isolation of existing certified designs, and isolation of major systems or equipment.

12-2 Development of a report describing the proposed approaches in detail.

\subsection{Challenges and Considerations Related to Vertical Loading of Certified Designs with Base Isolation Systems}

As discussed above, SI can significantly reduce the horizontal response of an isolated superstructure to incoming seismic motions. The properties of a base isolation system can be specified such that the incoming horizontal shaking level is reduced to below the SI-CSDRS. However, base isolation as commonly applied cannot be used to modify vertical motions entering the superstructure. Further, the typical tools for determining $\mathrm{V} / \mathrm{H}$ ratios are inappropriate for defining the design spectra of seismically-isolated super structures. For this reason, designers of seismically-isolated NPPs need to consider carefully the vertical spectrum that is used for a certified design. Although it has not been considered to date, one approach may be to evaluate carefully the equipment within an NPP design that may be sensitive to vertical motions and to develop not one, but a set of certified designs that each utilize the same horizontal design spectra but different vertical design spectrum.

Consideration should also be given to exploration of newly developed vertical isolation systems, although the challenges related to applying technologies that do not have the same extensive history of use as rubber and FP bearings require additional activities prior to their use. Chapter 12 of ASCE/SEI 4-16 and the draft SI NUREG/CR report provide a protocol for demonstrating that a new isolation technology is appropriate and that its safety can be assessed.

Topic 13, identification of challenges posed by vertical loading, was determined to be a mediumpriority, medium-term effort. The following technology R\&D activity would support commercial deployment of SI technologies at advanced reactors. To enable widespread and speedy dissemination, all research-oriented products would be made available via the INL Seismic Research Group website identified previously.

13-1 Identify issues related to the increase of the $\mathrm{V} / \mathrm{H}$ ratio during the design and licensing processes.

\subsection{Clarification of Legacy Terminology}

\subsubsection{Foundation}

As discussed in more detail in INL/EXT-15-36945, Section 3.4.2, the definition of the word "foundation," as applied to an NPP facility was historically relatively straightforward because the foundation was essentially a monolithic surface-founded or near-surface-founded structure that moved as a single structural element when subjected to seismic loading. When considering an isolated facility, the meaning or interpretation of the word "foundation," as written in the CFR or regulatory guidance documents, may not be straightforward.

ASCE/SEI 4-16 and the draft NUREG/CR report provide definitions that describe and clarify the terminology used in those guidance documents. However, the definition of "foundation," as defined in ASCE/SEI 4-16 and the draft SI NUREG/CR report, cannot be blindly applied retroactively for 
interpreting the meaning of "foundation" as used in in the CFR and other existing guidance documents. The term "foundation," in existing guidance, may apply to the foundation (which comprises those elements in contact with the ground), the upper basemat (which directly supports the superstructure), or the entire isolation system (which includes the foundation, the pedestals, the isolation layer, and the upper basemat). In some deeply-embedded advanced reactors, other conflicts may arise. The efficient and timely licensing of advanced reactors would benefit from activities to clarify the use of the term foundation in legacy documentation.

An example of the ambiguity that can arise around the term foundation is found in INL/EXT-15-36945, Section 3.5, which provides a detailed discussion of a resulting challenge related to application of the $0.1 \mathrm{~g}$ minimum spectrum described in Section IV(a)(1)(i) of 10 CFR 50, Appendix S. This requirement is addressed for conventionally-founded structures in the Standard Review Plan (SRP), Section 3.7.1, and DC/COL-ISG-017. In conventionally-founded structures, the free field surface response can be tied directly to the motions going into the superstructure and the safety-related equipment. However, there may be ambiguity as to where the minimum motions should be applied in a base-isolated NPP (i.e., at the foundation of the structure or at the upper basemat).

Topic 14, clarification of legacy terminology, was determined to be a high-priority, near-term effort. The following R\&D activities are needed to support commercial deployment of SI technologies at advanced reactors. To enable widespread and speedy dissemination, all research-oriented products would be made available via the INL Seismic Research Group website identified previously.

14-1 Identify legacy words in key regulatory documents, determine the function, and map to current terminology. This should include clarification of the location of the $0.1 \mathrm{~g}$ minimum spectrum.

14-2 Summarize findings report in support of licensing of advanced reactors and reactors using SI.

\subsubsection{Design Basis Earthquake}

Section 3.4.1 of INL/EXT-15-36945 discussed the use of the legacy term "design basis earthquake" as a regulatory challenge. Originally, the ground motions determined from the hazard studies were used directly as the design input ground motions for the facility. Therefore, the site-specific ground motions (developed by combining a site-specific PGA with a standard spectrum) were associated directly with the site-specific design. More recent approaches do not necessarily use site-specific ground motion directly in design. For example, NPPs designed and licensed using the certified design process detailed in 10 CFR 52 were designed primarily to site-independent CSDRS.

To address this disconnect in terminology, the term GMRS was implemented to describe site-specific motions when the NRC developed RG 1.208 (NRC 2007). The term Safe Shutdown Earthquake continues to be used for the motions used for design and licensing. Thus, the use of the term Design Basis Earthquake is no longer routinely found in guidance documents related to new reactors.

As a result, during its review, the INL Seismic Advisory Panel recommended that this topic (Topic 15) be given a low priority. Based on reviewer input, efforts on this topic should be limited to developing a short write-up of the background of the term to be included in the report for Topic 14, as discussed above.

15-1 Produce short write-up on the background of the term Design Basis Earthquake and include it in report for Topic 14, as appropriate. 


\section{CONFIGURATIONS AND APPLICATIONS NOT ADDRESSED IN FORTHCOMING GUIDANCE}

\subsection{Use of Isolators Exposed to Radiation and Other Environmental Conditions Uncommon to Seismic Isolation Systems}

As discussed in INL/EXT-15-36945, Section 4.1, the draft SI NUREG/CR report and the mandatory language for SI in ASCE/SEI 4-16 are written for reactor designs in which the isolators are protected from exposure to both high-radiation dosage and extreme temperatures. These limitations were imposed to be consistent with the exiting global SI experience and knowledgebase. However, these limitations constrain the possible applications and benefits of utilizing SI technology.

Although it is a frequent point of discussion, the change in mechanical properties of elastomeric and sliding isolators installed where radiation significantly exceeds that occurring naturally in the environment has not been established by either first-principles chemistry calculations or numerical and physical experiments. Such calculations and experiments on a family of elastomers and composites would be required to support the application of SI in a high radiation environment. Cladding of contemporary isolators with perimeter radiation-hardened materials (i.e., similar to cover rubber used on elastomeric isolators to provide protection from environmental effects) may provide a viable alternative worthy of calculations and experiments.

The mechanical properties of elastomeric and sliding isolation bearings are generally selected and quantified by testing for ambient conditions of pressure and temperature at sea level. Isolators expected to operate at elevated (or significantly lower) temperatures for decades may require the development of specially compounded elastomers and composites, supported by first-principles calculations and numerical and physical experiments. Similar activities related to humidity and reactive or corrosive environments may benefit some applications.

The INL Seismic Advisory Committee identified the investigation on the use of isolators in new environmental conditions (Topic 16) as a low priority effort with a scope or work and timeline to be determined based on the identification of industry partner with whom to work. To enable widespread dissemination, all research-oriented products would be made available via the INL Seismic Research Group website identified previously.

16-1 With industry partner, investigate the use of isolators in new environmental conditions.

\subsection{Isolation of Components, Equipment, and Distributed Systems}

As discussed in INL/EXT-15-36945, Section 4.2, the draft SI NUREG/CR report does not discuss the isolation of components, equipment, and distributed systems in any detail. The Seismic Advisory Committee considered this to be a significant gap for the use of SI in advanced reactors. Thus, a new section (Section 2.2) was added to develop and documents methods for incorporating equipment isolation in advanced reactors, and other sections (2.4.2 and 3.3) were expanded to more fully address isolation of components. Discussions and tasks can be found in those sections.

No specific tasks related to distributed systems were identified at this time. 


\section{SEISMIC QUALIFICATION OF ISOLATOR UNITS}

One identified challenge related to the licensing of isolated NPPs involves identifying and justifying the most appropriate and achievable approach to seismic qualification of the isolators. Recent work in industry has determined that the commercial grade dedication (CGD) process, described in EPRI (2014), is an appropriate equivalent approach that is well suited to addressing the needs of isolator units.

INL/EXT-15-36945, Section 5, discussed the CGD process and its application to SI units in detail. The reader is referred to that document for more information on the process, testing requirements, documentation requirements, and other aspects. Although the application of the process requires little additional technical basis development, the timely and efficient licensing of advanced reactors using SI would benefit from the development of detailed written guidance on the application of the CGD process to SI. This documentation should address the approach to meeting testing performance requirements described in the draft NUREG/CR. ${ }^{14}$

Topic 17, documentation of the use of the CGD process as an appropriate equivalent approach to seismic qualification of isolator units, was determined to be a medium-priority, short-term effort. The following R\&D activity would reduce regulatory risk related to use of SI technologies at advanced reactors. To enable widespread and speedy dissemination, all research-oriented products would be made available via the INL Seismic Research Group website identified previously.

17-1 Develop a guidance document on the application of the CDG process as an appropriate equivalent approach to the qualification of seismic isolator units. Develop a publicly available report to support discussions and agreement of the questions about use of the CDG process.

14 As noted in Section 5.2 of INL/EXT-15-36945. 


\section{LICENSING COMMITMENTS FOR CONSTRUCTION AND OPERATIONS}

\subsection{Considerations for Inspection, Testing, Analysis, and Acceptance Criteria}

In INL report INL/EXT-14-33234 (Coleman and Sabharwall 2014), appropriate development of inspection, testing, analysis, and acceptance criteria (ITAAC) is noted as a regulatory consideration (and potential issue) for facilities using SI technology. As described in INL/EXT-15-36945, Section 6, the ITAAC are detailed conditions to a combined license issued by the NRC.

The draft SI NUREG/CR report addresses several elements that should be considered for inclusion in the ITAAC. These SI-related considerations for the ITAAC include the isolator testing program, additional seismic monitoring equipment, tilt meters, umbilical lines and other crossover SSCs, and additional performance-based criteria for deformation of the foundation and basemat. These items are discussed in detail in INL/EXT-15-36945, Section 6.1.

Because an NPP utilizing SI has not been licensed in the U.S. to date, the ITAAC for such a facility has not been developed. This section provides information on elements that have been identified for inclusion into the ITAAC. However, the necessary licensing submittal would require far more technical detail than is provided above. Although some of the information in the ITAAC will be facility-specific, a significant portion of the necessary documentation could be developed in the short-term using available information and focusing on the three elements of the ITAAC: design commitments; inspections, tests and analyses; and acceptance criteria. Development of a report with draft documentation would benefit the efficient and timely licensing of advanced reactors using SI.

Topic 18, which focuses on developing guidance on the ITAAC for licensing isolated facilities, was determined to be a high-priority, short-term effort. The following R\&D activity is needed to reduce regulatory risk related to use of SI technologies at advanced reactors. To enable widespread and speedy dissemination, all research-oriented products would be made available via the INL Seismic Research Group website identified previously.

18-1 Develop a guidance document on development of the SI-related ITACC for advanced reactors. This should include minimum requirements for inclusion in ITAAC for a new isolated facility focusing design commitment; inspections, tests, and analyses; and acceptance criteria.

\subsection{In-Service Inspections, Testing, and Operations}

The in-service inspection and testing program must be defined as part of the license application. The program should provide complete information on the method of inspection and testing, the acceptance criteria, and the steps to be taken if the inspection or testing indicates that the isolators or isolation system does not continue to meet the requirements. Section 6.2 of INL/EXT-15-36945 provides an in-depth discussion of the following elements:

- In-service testing

- In-service visual inspection

- In-service monitoring of the foundation and moat

- Post-earthquake inspection

- Isolator unit removal and replacement requirements. 
Although many items related to in-service inspections, in-service testing, and operational considerations have been identified and discussed in INL/EXT-15-36945, the complete treatment of these elements in the regulatory process (and a proposed best practice approach) have not been fully investigated and documented. An effort focused in this area could be conducted in the short-term and would not be expected to involve a significant level of additional research. This effort would support the efficient and timely licensing of advanced reactors using SI.

Topic 19, which focused on developing guidance on the in-service inspections, testing and operational requirements for licensing isolated facilities, was determined to be a high-priority, short-term effort. The following R\&D activity is needed to reduce regulatory risk related to use of SI technologies at advanced reactors. To enable widespread and speedy dissemination, all research-oriented products would be made available via the INL Seismic Research Group website identified previously.

19-1 Develop a guidance document on development of the minimum standards for in-service inspections, testing, and operations of advanced reactors using SI technology. This report should include information related to both base isolation and equipment isolation. 


\section{IDENTIFIED ISSUES AND PROPOSED RESOLUTIONS}

This report identifies and discusses regulatory guidance gaps and challenges for the licensing of NPPs using SI technologies, including isolated advanced reactor designs. As an aid to the reader, Table 2 summarizes the gaps and challenges that were identified in prior sections of this report, and the identified approaches for addressing each topic. The "Importance" indicator in the table relates to the degree to which the issue may impact ability to efficiently license advanced reactors with seismic isolation in a timely manner; the designators are self-explanatory.

Table 2 includes information on whether the timeframe to complete the activities described in the path forward is a short-, medium-, or long-term effort, where short term is 1-3 years, medium term is 35 years, and long term is $5-10$ years. The actions that have been identified as the most high-impact and/or high-value topics in terms of their degree of support for licensing activities are highlighted orange in Table 2.

The gaps and challenges identified are summarized in Table 2. The 19 unique gaps and challenges identified and evaluated have each been assigned a Topic number; and proposed actions are assigned an associated task number. Table 2 provides a summary roadmap of identified tasks, including possible timelines. Based on the roadmap provided, it is recommended that four Topics should be prioritized for funding:

- Development of NLSSI tools and guidance, including verification and validation activities. (Topic 1)

- Development of SPRA methodologies for seismically isolated facilities, with an emphasis on advancement of methods applicable when linear scaling assumptions do not apply. (Topic 6)

- Clarification of the approaches to licensing of facilities using seismic isolation technology within the certified design process and the associated clarification of the intent of the term "foundation" in existing requirements and guidance (Topics 12 and 14)

- Development of approaches and guidance for isolation of large equipment (Topic 4)

One DOE goal specified in the draft document entitled, "Vision and Strategy for the Development and Deployment of Advanced Reactors," $" 15$ is:

\section{"By the early 2030s, at least two non-light water advanced reactor concepts would have reached technical maturity, demonstrated safety and economic benefits, and completed licensing reviews by the U.S. Nuclear Regulatory Commission (NRC) sufficient to allow construction to go forward."}

SI has been identified as an important technology that both facilitates the siting of advanced reactors in a wider range of locations and improves seismic safety. SI can also minimize one off advanced reactor designs that would otherwise need to be changed to meet site specific seismic hazard curves.

Minimization of "one-off" designs should improve economics of advanced reactors. Several of the topics and tasks identified in this report support the design and licensing of advanced reactors, regardless of whether SI technology is used, particularly if the NPPs are deeply embedded. Additionally, current advanced reactor venders, in collaboration with INL, are interested in initiating sensitivity studies for seismic isolation of their specific technologies. This report provides a 10-year timeline for completing activities to address the issues identified. Not starting the four near term activities in a timely way would limit the impact of the collaborative work with advanced reactor venders due to the regulatory

\footnotetext{
${ }^{15}$ The 27 May 2016 version of this document is available for download at https://www.energy.gov/ne/downloads/draft-visionand-strategy-development-and-deployment-advanced-reactors.
} 
uncertainty. A delayed start, as well as a lengthened timeline due to insufficient funding, would also make achieving the goals laid out in the DOE vision document less likely. 
Table 2. Summary of identified topics, importance, and proposed resolutions.

\begin{tabular}{|c|c|c|c|c|c|c|c|c|c|c|c|c|c|c|c|c|}
\hline$\Xi$ & & & & & & & & & & & & & & & & \\
\hline$a$ & & & & & & & & & & & & & & & & \\
\hline$\infty$ & & & & & & & & & & & & & & & & \\
\hline$n$ & & & & & & & & & & & & & & & & \\
\hline 6 & & & & & & & & & & & & & & & & \\
\hline is & & & & & & & & & & & & & & & & \\
\hline+ & & & & & & & & & & & & & & & & \\
\hline$m$ & & & & & & & & & & & & & & & & \\
\hline$N$ & & & & & & & & & & & & & & & & \\
\hline- & & & & & & & & & & & & & & & & \\
\hline 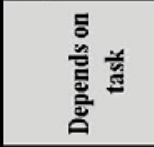 & 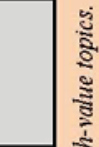 & & $I$ & & $\stackrel{m}{I}$ & $\begin{array}{l}\frac{I}{I} \\
\Xi \\
I \\
I\end{array}$ & & 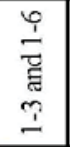 & & 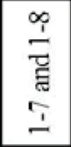 & & & 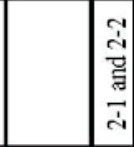 & 彳 & & $\begin{array}{l}\text { v } \\
\dot{m} \\
\text { है } \\
\dot{m}\end{array}$ \\
\hline 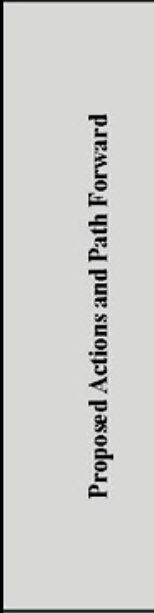 & 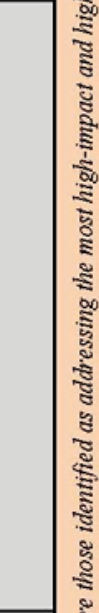 & 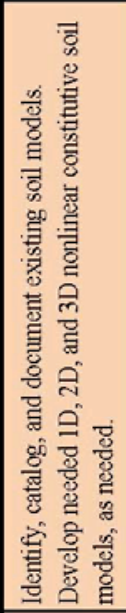 & 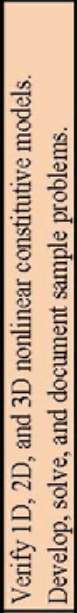 & 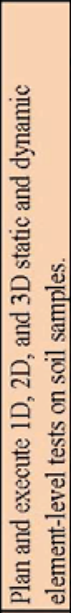 & 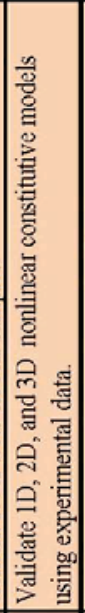 & 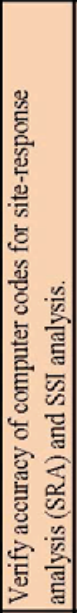 & 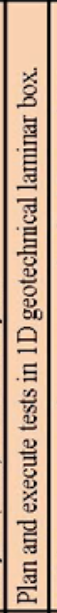 & 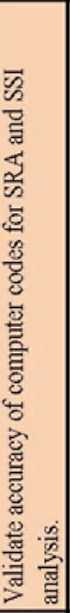 & 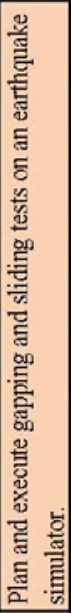 & 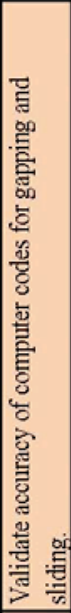 & 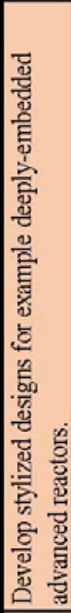 & 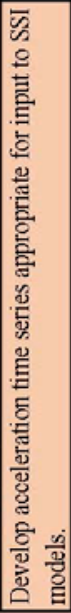 & 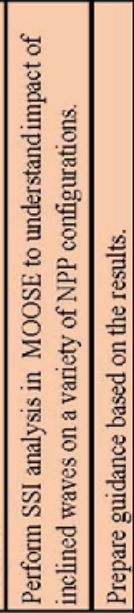 & 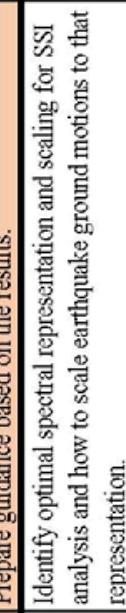 & 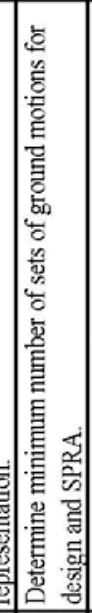 & 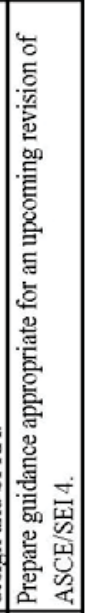 \\
\hline 㟔 产 & 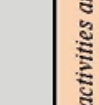 & 吾 & 害 & $\frac{\underline{t}}{\sqrt{2}}$ & 嘉 & $\begin{array}{l}\text { 焉 } \\
\frac{\pi}{2}\end{array}$ & 홀 & $\begin{array}{l}\text { 悬 } \\
\text { 恶 }\end{array}$ & 总 & 害 & & 县 & & $\begin{array}{l}\text { 吾 } \\
\text { 恶 }\end{array}$ & 喜 & 主 \\
\hline 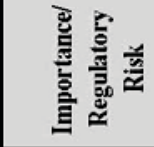 & 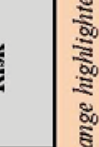 & 点 & 矛 & 点 & 总 & 总 & 哥 & 总 & 总 & 总 & & 象 & & 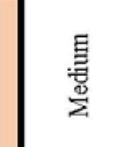 & 憘 & $\begin{array}{l}\text { 基 } \\
\frac{\mathrm{e}}{2}\end{array}$ \\
\hline 奠 & & $\Xi$ & $I$ & $\stackrel{m}{I}$ & \pm & $\because$ & $\stackrel{\circ}{-}$ & $I$ & $\stackrel{\infty}{\perp}$ & $\stackrel{i}{i}$ & $\bar{\lambda}$ & ปี & $\dot{\sim} \vec{c}$ & $\overrightarrow{~ \vec{~}}$ & rூ & $\dot{m}$ \\
\hline 言完 & & & & & - & & & & & & & c & & & m & \\
\hline 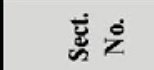 & & & & & $\exists$ & & & & & & & ? & & & $\frac{m}{i}$ & \\
\hline$\underline{2}$ & & & & & 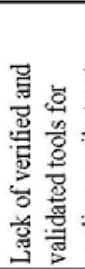 & 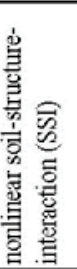 & & & & & & 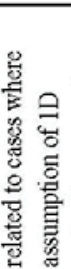 & 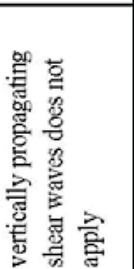 & & 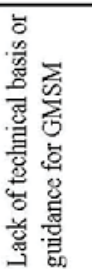 & \\
\hline
\end{tabular}


Table 2. Cont.

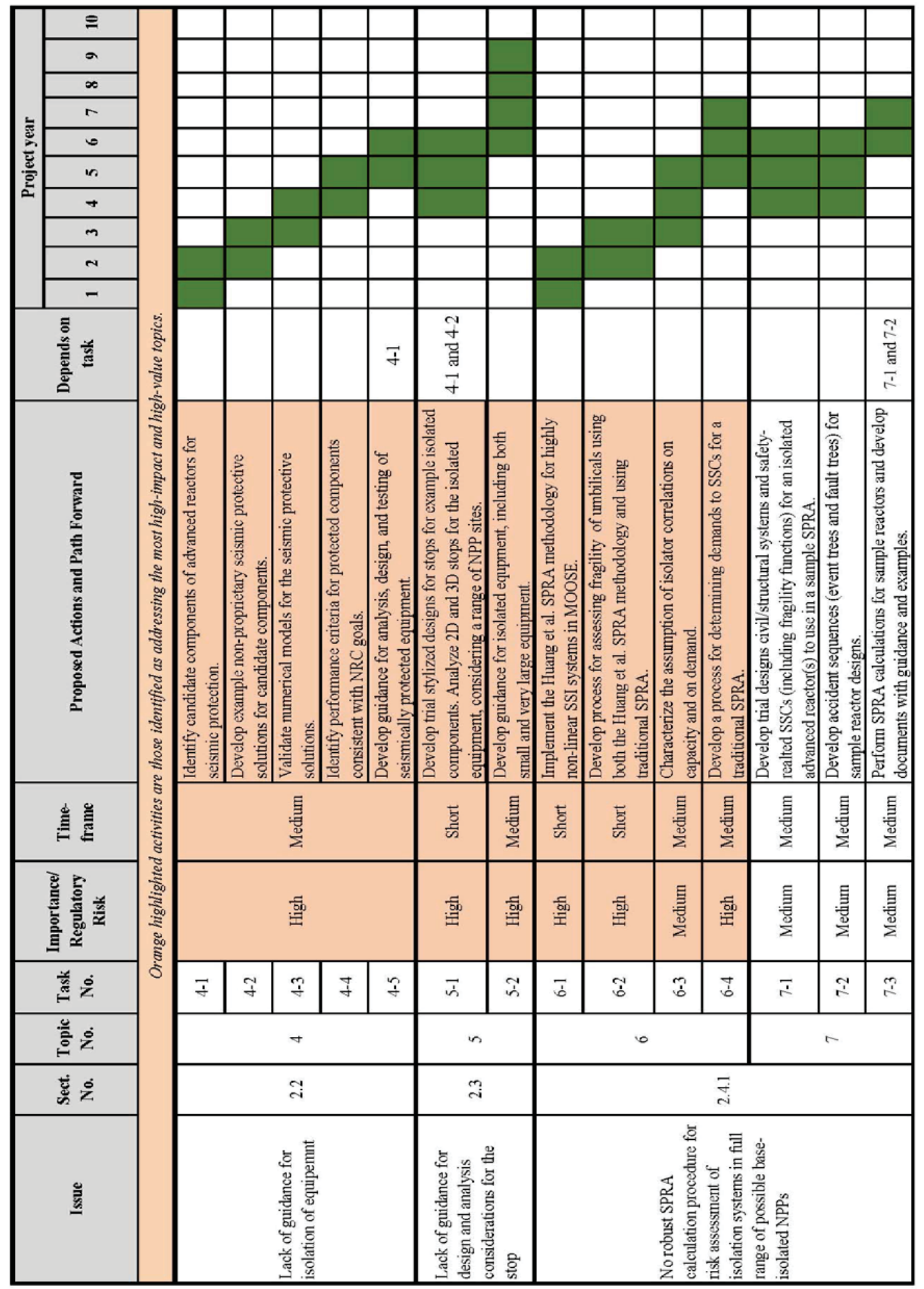


Table 2. Cont.

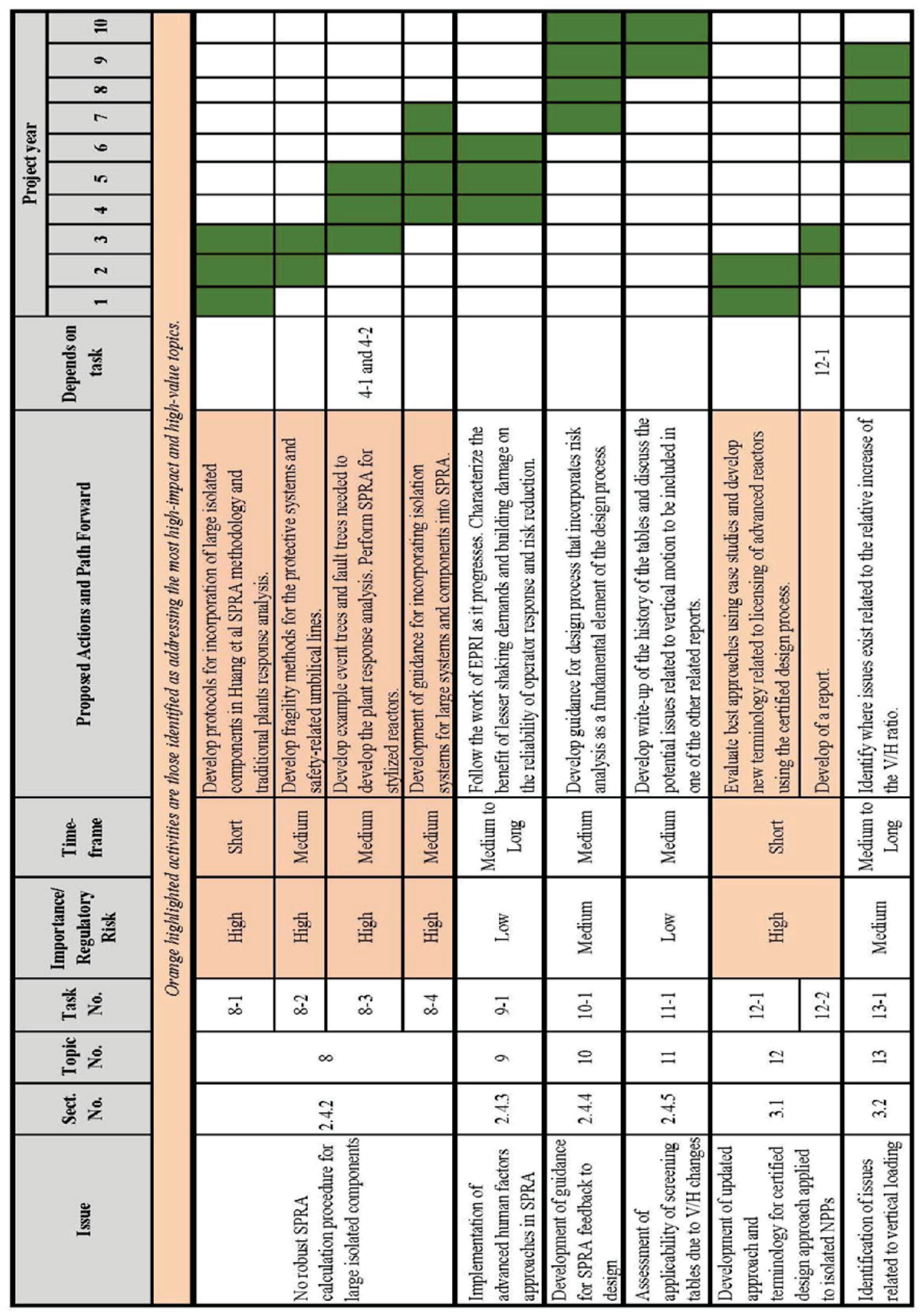


Table 2. Cont.

\begin{tabular}{|c|c|c|c|c|c|c|c|c|c|}
\hline & 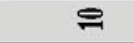 & & & & & & & & \\
\hline & $a$ & & & & & & & & \\
\hline & $\infty$ & & & & & & & & \\
\hline & $r$ & & & & & & & & \\
\hline$\Xi$ & 6 & & & & & & & & \\
\hline ฮ & in & & & & & & & & \\
\hline & $\vec{\sigma}$ & & & & & & & & \\
\hline & 9 & & & & & & & & \\
\hline & $\sim$ & & & & & & & & \\
\hline & - & & & & & & & & \\
\hline & 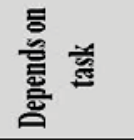 & 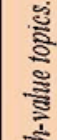 & & 主 & & & & & \\
\hline & 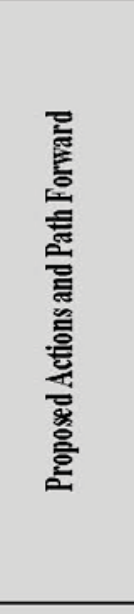 & 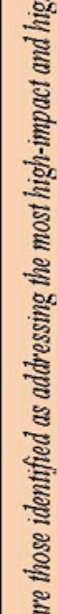 & 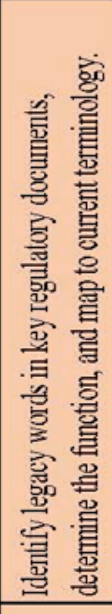 & 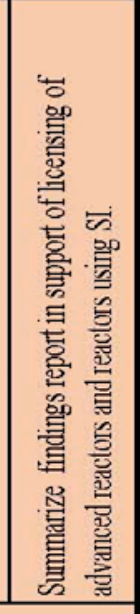 & 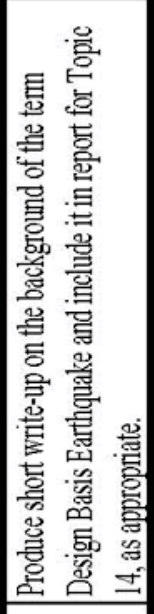 & 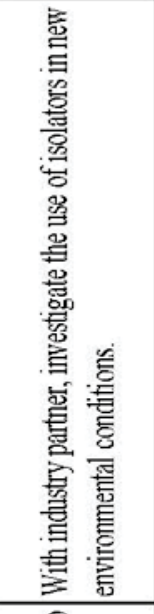 & 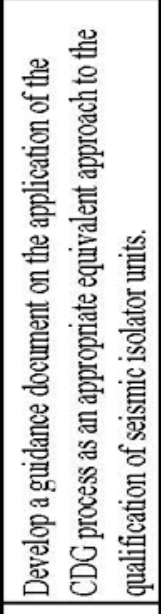 & 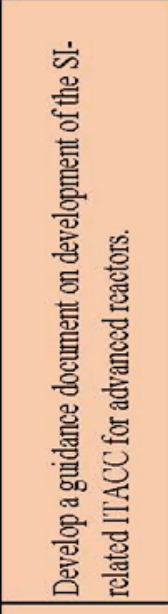 & 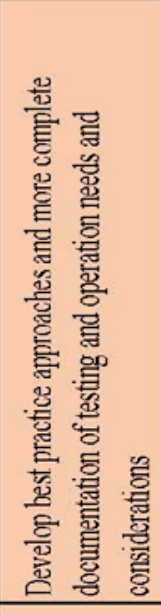 \\
\hline & 咅竎 & हू & & 홀 & 壳 & $\begin{array}{l}\text { 总 } \\
\text { 喜 } \\
\text { 兽 }\end{array}$ & 홇 & 홇 & 点 \\
\hline & 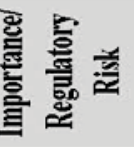 & 咅 & & 营 & 危 & 客 & $\begin{array}{l}\text { 喜 } \\
\text { 尊 }\end{array}$ & 嚁 & 请 \\
\hline & 曾 & & I & I & $\vec{\Xi}$ & $\overrightarrow{\dot{b}}$ & $\Xi$ & $\vec{\infty}$ & $\vec{a}$ \\
\hline & 产 을 & & & $\Xi$ & $\because$ & 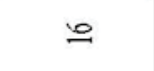 & $\Xi$ & 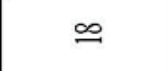 & 으 \\
\hline & 总 & & & $m$ & & $\rightleftarrows$ & $n$ & $\overrightarrow{6}$ & 뭉 \\
\hline & 夠 & & & 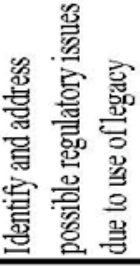 & $\begin{array}{l}\text { 离 } \\
\text { 高 } \\
\text { 息 } \\
\end{array}$ & 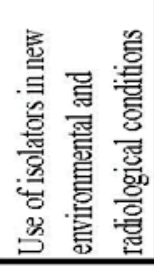 & 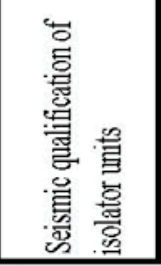 & 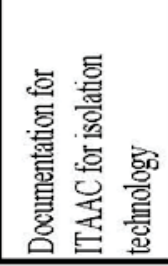 & 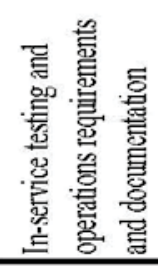 \\
\hline
\end{tabular}




\section{REFERENCES}

10 CFR 50, "Domestic Licensing of Production and Utilization Facilities."

10 CFR 52, "Licenses, Certifications, and Approvals for Nuclear Power Plants."

ANSYS Inc., 2013, “Computer program ANSYS 13.0,” Canonsburg, Pennsylvania.

ASCE, 2005. ASCE/SEI 43-05, "Seismic design criteria for structures, systems and components in nuclear facilities," Structural Engineering Institute of the American Society of Civil Engineers, Reston, Virginia.

ASCE/SEI, 2016, "Seismic analysis of safety-related nuclear structures," ASCE/SEI 4-16, Structural Engineering Institute of the American Society of Civil Engineers, Reston, Virginia.

ASME, 2006, "Verification and validation in computational solid mechanics," ASME VV-10, American Society of Mechanical Engineers, New York, New York.

ASME, 2015, "Quality assurance requirements for nuclear facility applications," NQA-1-2015, American Society of Mechanical Engineers, New York, New York.

ASME/ANS, 2013, "Probabilistic risk assessment standard for advanced non-LWR nuclear power plants," RA-S-1.4-2013, American Society of Mechanical Engineers/American Nuclear Society, New York, New York.

Baker, J. W., and C. A. Cornell, 2006, "Spectral shape, epsilon and record selection," Earthquake Engineering and Structural Dynamics, Vol. 35, No. 9, pp. 1077-1095.

Bielak, J., 1975, "Dynamic behavior of structures with embedded foundations," Earthquake Engineering and Structural Dynamics, Vol. 3, pp. 259-274.

Bielak, J., and P. Christiano, 1984, "On the effective seismic input for nonlinear soil-structure interaction systems," Earthquake Engineering and Structural Dynamics, Vol. 12, No. 1, pp. 107-119.

Bielak, J., K. Loukakis, Y. Hisada, and C. Yoshimura, 2003, "Domain reduction method for three-dimensional earthquake modeling in localized regions, Part-I: theory," Bulletin of the Seismological Society of America, Vol. 93, No. 2, pp. 817-824.

BNL, 2009, Consistent site response-SSI calculations, Report N6112-051208, Rev. 1, Brookhaven National Laboratory, Stonybrook, New York (ADAMS Accession No. ML091980384).

Bolisetti, C., and A. S. Whittaker, 2015, Site Response, Soil-Structure Interaction, and Structure-Soil-Structure Interaction for Performance Assessment of Buildings and Nuclear Structures, Technical Report MCEER-15-0002, University at Buffalo, State University of New York, Buffalo, New York.

Bolisetti, C., A. S. Whittaker, and J. Coleman, 2016, "Linear and nonlinear soil-structure-interaction analysis," submitted to Soil Dynamics and Earthquake Engineering.

Bolisetti, C., A. S. Whittaker, H. B. Mason, I. Almufti, and M. Willford, 2014, "Equivalent linear and nonlinear site-response analysis for design and risk assessment of safety-related nuclear structures," Nuclear Engineering and Design, Vol. 275, pp. 107-121, http://dx.doi.org/10.1016/j.nucengdes.2014.04.033.

Bolisetti, C., J. L. Coleman, M. Talaat, and P. Hashimoto, 2015, Advanced Seismic Fragility Modeling using Nonlinear Soil-Structure Interaction Analysis, INL/EXT-15-36735, Idaho National Laboratory, Idaho Falls, Idaho. 
Budnitz, R. J. and M. W. Mieler, 2016, Toward a more risk-informed and performance-based framework for the regulation of the seismic safety of nuclear power plants, NUREG/CR-7214, United States Nuclear Regulatory Commission, Washington, D.C.

Coleman, J., and P. Sabharwall, 2014, Seismic Isolation Working Meeting Gap Analysis Report Development, INL/EXT-14-33234, Idaho National Laboratory, Idaho Falls, Idaho.

Coleman, J., C. Bolisetti, and A. S. Whittaker, 2016a, "Time-domain soil-structure interaction analysis of nuclear facilities," Nuclear Engineering and Design, Vol. 298, March 2016, pp. 264-270

Coleman, J. L., C. Bolisetti, S. Veeraraghavan, C. Parisi, S. R. Prescott, A. Gupta, and A. M. Kammerer, 2016b, Multi-Hazard Advanced Seismic Probabilistic Risk Assessment Tools and Applications, INL/EXT-16-40055, Idaho National Laboratory, Idaho Falls, Idaho.

Dassault Systèmes, 2015, “Computer program ABAQUS,” Providence, Rhode Island.

DOE, 2002, DOE-STD-1020-2002, "Natural phenomena hazards design and evaluation criteria for Department of Energy facilities," United States Department of Energy, Washington, D.C.

EPRI, 1991, A Methodology for Assessment of Nuclear Power Plant Seismic Margin, Report NP-6041-SLR1, Rev 1, Electric Power Research Institute, Palo Alto, California.

EPRI, 2014, Plant Engineering: Guideline for the Acceptance of Commercial-Grade Items in Nuclear Safety-Related Applications: Revision 1 to EPRI NP-5652 and TR-102260, Report 3002002982, Electric Power Research Institute, Palo Alto, California.

FEMA, 2012, Seismic Performance Assessment of Buildings, Volume 1-Methodology, FEMA P-58-1, Federal Emergency Management Agency, Washington, D.C.

Huang, Y.-N., A. S. Whittaker, and N. Luco, 2008a, "Maximum spectral demands in the near-fault region," Earthquake Spectra, Vol. 24, No. 1, pp. 319-341.

Huang, Y.-N., A. S. Whittaker, and N. Luco, 2008b, "Performance assessment of conventional and base-isolated nuclear power plants for earthquake and blast loadings," Technical Report MCEER-08-0019, University at Buffalo, State University of New York, Buffalo, New York.

Huang, Y.-N., A. S. Whittaker, and N. Luco, 2009a, "Orientation of maximum spectral demand in the near-fault region," Earthquake Spectra, Vol. 25, No. 3, pp. 707-717.

Huang, Y.-N., A. S. Whittaker, and N. Luco. 2011a, "A seismic risk assessment procedure for nuclear power plants, (I) methodology," Nuclear Engineering and Design, Vol. 241, pp. 3996-4003.

Huang, Y.-N., A. S. Whittaker, and N. Luco, 2011b, “A seismic risk assessment procedure for nuclear power plants, (II) application," Nuclear Engineering and Design, Vol. 241, pp. 4004-4011.

Huang, Y.-N., A. S. Whittaker, R. P. Kennedy, and R. L. Mayes, 2009b, “Assessment of base-isolated nuclear structures for design and beyond-design-basis earthquake shaking," Technical Report MCEER-09-0008, University at Buffalo, State University of New York, Buffalo, New York.

Huang, Y.-N., A. S. Whittaker, R. P. Kennedy, and R. L. Mayes, 2013, "Response of base-isolated nuclear structures for design and beyond-design basis earthquake shaking," Earthquake Engineering and Structural Dynamics, Vol. 42, No. 3, pp. 339-356.

INL, 2011. Modular HTGR safety basis and approach, INL/EXT-11-22708, Idaho National Laboratory, Idaho Falls, Idaho.

INL, 2014, Guidance for developing principal design criteria for advanced (non-light water) reactors, INL/EXT-14-31179, Revision 1, Idaho National Laboratory, Idaho Falls, Idaho. 
Jayaram, N., T. Lin, and J. W. Baker, 2011, “A computationally efficient ground-motion selection algorithm for matching a target response spectrum mean and variance," Earthquake Spectra, Vol. 27, No. 3, pp. 797-815.

Jeremić, B., G. Jie, M. Preisig, and N. Tafazzoli, 2009, "Time domain simulation of soil-foundationstructure interaction in non-uniform soils," Earthquake Engineering and Structural Dynamics, Vol. 38, No. 5, pp. 699-718.

Jeremić, B., R. Roche-Rivera, A. M. Kammerer, N. Tafazzoli, J. Abell, M. B. Kamrani-moghaddam, F. Pisano, C. Jeong, and B. Aldridge, 2013, "The NRC ESSI simulator program," Transactions, 22nd International Conference in Structural Mechanics in Reactor Technology (SMiRT), San Francisco, California.

Kammerer, A., A. Whittaker, and J. Coleman, 2016, Regulatory gaps and challenges for licensing advanced reactors using seismic isolation, INL/EXT-15-36945, Idaho National Laboratory, Idaho Falls, Idaho.

Kammerer, A.M., A. S. Whittaker, and M. C. Constantinou, 2017, Technical considerations for the seismic isolation of nuclear facilities, NUREG/CR-XXXX, United States Nuclear Regulatory Commission, Washington, D.C. (in publication)

Kammerer, A., J. M. Pestana, and R. B. Seed, 2002, "Undrained response of Monterey 0/30 sand under multidirectional cyclic simple shear loading conditions," Technical Report UCB/GT/02-01, Department of Civil and Environmental Engineering, University of California, Berkeley, California.

Kumar, M., and A. S. Whittaker, 2016, "Seismic probabilistic risk assessment of safety-related nuclear facilities," submitted to Nuclear Engineering and Design.

Kumar, M., A. S. Whittaker, and M. C. Constantinou, 2014, "An advanced numerical model of elastomeric seismic isolation bearings," Earthquake Engineering and Structural Dynamics, Vol. 43, No. 13, pp. 1955-1974.

Kumar, M., A. S. Whittaker, and M. C. Constantinou, 2015a, "Characterizing friction in sliding isolation bearings," Earthquake Engineering and Structural Dynamics, Vol. 44, No. 9, pp. 1409-1425, July 2015.

Kumar, M., A. S. Whittaker, and M. C. Constantinou, 2015b, "Seismic isolation of nuclear power plants with sliding bearings," Technical Report MCEER-15-0006, University at Buffalo, State University of New York, Buffalo, New York

Kumar, M., A. S. Whittaker, and M. C. Constantinou, 2015c, "Seismic isolation of nuclear power plants with elastomeric bearings," Technical Report MCEER-15-0008, University at Buffalo, State University of New York, Buffalo, New York.

LSTC, 2013, “Computer program LS-DYNA,” Version 7.0, Livermore Software Technology Corporation, Livermore, California.

Luco, J. E., and L. Contesse, 1973, "Dynamic structure-soil-structure interaction," Bulletin of the Seismological Society of America, Vol. 63, No. 4, pp. 1289-1303.

Luco, J. E., and R. A. Westman, 1971, "Dynamic response of circular footings," Journal of Engineering Mechanics Division, Vol. 97, EM5, pp. 1381-1395.

Lysmer, J., and R. L. Kuhlemeyer, 1969, "Finite dynamic model for infinite media," Journal of Engineering Mechanics Division, Vol. 95, EM4, pp. 859-877.

Lysmer, J., F. Ostadan, and C. Chin, 1999, "Computer program SASSI2000--a system for analysis of soil-structure interaction," University of California, Berkeley, California. 
Mason, H. B., 2011, "Seismic performance assessment in dense urban environments," Ph.D. dissertation, University of California, Berkeley, California.

Mathworks, 2016, “Computer program MATLAB," Natick, Massachusetts.

Mazzoni, S., F. McKenna, M. H. Scott, and G. L. Fenves, 2009, “Computer program OpenSees: Open System for Earthquake Engineering Simulation," Pacific Earthquake Engineering Research Center, University of California, Berkeley, California.

NEI, 2009, "Consistent site-Response/Soil-Structure Interaction Analysis and Evaluation," Nuclear Energy Institute, Washington, D.C. (ADAMS Accession No. ML091680715).

NIST, 2011, "Selection and Scaling of Earthquake Ground Motions for Performing Response-History Analysis," Technical Report NIST GCR 11-917-15, National Institute of Standards and Technology, Gaithersburg, MD.

NRC, 2007a, "Standard Review Plan for the Review of Safety Analysis Reports for Nuclear Power Plants," NUREG-0800, United States Nuclear Regulatory Commission, Washington, D.C. (ADAMS Accession Nos. ML070730593, ML070640306, ML070640311).

NRC, 2007b, "A Performance-Based Approach to Define the Site-Specific Earthquake Ground Motion," Regulatory Guide 1.208, United States Nuclear Regulatory Commission, Washington, D.C. (ADAMS Accession No. ML070310619).

NRC, 2007c, "Seismic Design Classification," Regulatory Guide 1.29, Revision 4, United States Nuclear Regulatory Commission, Washington, D.C. (ADAMS Accession No. ML070310052).

NRC, 2009, "Interim Staff Guidance on Ensuring Hazard-Consistent Seismic Input for Site Response and Soil Structure Interaction Analyses," DC/COL-ISG-017, United States Nuclear Regulatory Commission, Washington, D.C. (ADAMS Accession No. ML100570203).

NRC, 2010a, "Interim Staff Guidance on Implementation of a Probabilistic Risk Assessment-Based Seismic Margin Analysis for New Reactors," DC/COL-ISG-020, United States Nuclear Regulatory Commission, Washington, D.C. (ADAMS Accession No. ML100491168).

NRC, 2010b, "Quality Assurance Program Criteria (Design and Construction)," Regulatory Guide 1.28, Rev. 4, United States Nuclear Regulatory Commission, Washington, D.C. (ADAMS Accession No. ML100160003).

NRC, 2012, "Practical Implementation Guidelines for SSHAC Level 3 and 4 Hazard Studies," NUREG2117, Rev. 1, Nuclear Regulatory Commission, Washington, D.C. (ADAMS Accession No. ML ML12118A445).

NRC, 2013, "Design-Specific Review Standard for mPOWER iPWR Design," United States Nuclear Regulatory Commission, Washington, D.C. (ADAMS Accession No. ML13099A205).

NRC, 2014, Regulatory Guide 1.60, "Design Response Spectra for Seismic Design of Nuclear Power Plants," Rev. 2, Nuclear Regulatory Commission, Washington, D.C.

Oberkampf, W. L., and C. J. Roy, 2010, Verification and validation in scientific computing, Cambridge University Press.

Ostadan, F., 2006a, "SASSI2000: a System for Analysis of Soil-Structure Interaction-User's Manual," University of California, Berkeley, California.

Ostadan, F., 2006b, "SASSI2000: a System for Analysis of Soil-Structure Interaction-Theoretical Manual," University of California, Berkeley, California. 
Ostadan, F., and N. Deng, 2011, “Computer Program SASSI2010,” Version 1.1, Geotechnical and Hydraulic Engineering Services, Bechtel National Inc., San Francisco, California.

Presley, M. R., J. A. Julius, J. F. Grobbelaar, and K. D. Kohlhepp, 2013a, “A preliminary approach to human reliability analysis for external events with a focus on seismic HRA." Proceedings, ANS PSA 2013 International Topical Meeting on Probabilistic Safety Assessment and Analysis, Columbia, South Carolina.

Presley, M. R., J. A. Julius, J. F. Grobbelaar, and K. D. Kohlhepp, 2013b, “A review of seismic operating experience with implications for human reliability," Proceedings, ANS PSA 2013 International Topical Meeting on Probabilistic Safety Assessment and Analysis, Columbia, South Carolina.

Ryan, H., 1994, "Ricker, Ormsby, Klander, Butterworth - a choice of wavelets," Canadian Society of Exploration Geophysicts Recorder, Vol. 19, No. 7, pp. 8-9.

Schnabel, P. B., J. Lysmer, and H. B. Seed, 1991, "Computer Program SHAKE: A Computer Program for Earthquake Response Analysis of Horizontally Layered Sites," University of California, Berkeley, California.

Seed, H. B., and I. M. Idriss, 1970, Soil Moduli and Damping Factors for Dynamic Response Analysis, Report UCB/EERC 70/10, Earthquake Engineering Research Center, University of California, Berkeley, California.

Taborda, R., and J. Bielak, 2011, "Large-scale earthquake simulation: computational seismology and complex engineering systems," Computing in Science and Engineering, Vol. 13, No. 4, pp. 14-27.

Veletsos, A. S., and B. Verbic, 1973, "Vibration of viscoelastic foundations," Earthquake Engineering and Structural Dynamics, Vol. 2, No. 1, pp. 87-105.

Veletsos, A. S., and J. W. Meek, 1974, "Dynamic behavior of building foundation systems," Earthquake Engineering and Structural Dynamics, Vol. 3, No. 2, pp. 121-138.

Veletsos, A. S., and Y. T. Wei, 1971, "Lateral and rocking vibration of footings," Journal of the ASCE Soil Mechanics and Foundations Division, Vol. 97, SM9, pp. 1227-1248.

Willford, M., R. Sturt, Y. Huang, I. Almufti, and X. Duan, 2010, "Recent Advances in Nonlinear Soil-Structure Interaction Analysis using LS-DYNA," Proceedings, NEA-SSI Workshop, Ottawa, Canada, October 6-8, 2010.

Wolf, J. P., 1985, Dynamic Soil-Structure Interaction, Prentice Hall, Englewood Cliffs, New Jersey.

Xu, J., J. Bielak, O. Ghattas, and J. Wang, 2003, “Three-dimensional nonlinear seismic ground motion modeling in basins," Physics of the Earth and Planetary Interiors, Vol. 137, pp. 81-95.

Yang, T. Y., J. P. Moehle, B. Stojadinovic, and A. Der Kiureghian, 2009, "Performance evaluation of structural systems: theory and implementation," Journal of Structural Engineering, Vol. 135, No. 10, pp. 1146-1154.

Yoshimura, C., J. Bielak, and Y. Hisada, 2003, "Domain reduction method for three-dimensional earthquake modeling in localized regions, part-II: verification and examples," Bulletin of the Seismological Society of America, Vol. 93, No. 2, pp. 825-840. 